\title{
Modeling and numerical approximation of two-phase incompressible flows by a phase-field approach
}

\author{
Jie Shen \\ Department of Mathematics, Purdue University \\ West Lafayette, IN 47907-1957, USA \\ shen@math.purdue.edu
}

\begin{abstract}
We present in this note a unified approach on how to design simple, efficient and energy stable time discretization schemes for the Allen-Cahn or Cahn-Hilliard Navier-Stokes system which models twophase incompressible flows with matching or non-matching density. Special emphasis is placed on designing schemes which only require solving linear systems at each time step while satisfy discrete energy laws that mimic the continuous energy laws. We construct the time discretization schemes in weak formulations so that they can be used with any consistent Galerkin type spacial discretization schemes such as finite element methods and spectral/spectral-element methods.
\end{abstract}

\section{Contents}

1 Introduction

2 phase-field model for two-phase incompressible and immiscible flows 2.1 The case of matched density

2.2 The case of variable density

3 Some efficient time discretization schemes for Navier-Stokes equations

3.1 Pressure-correction schemes

3.2 Pressure-stabilization schemes

4 Some efficient time discretization schemes for Allen-Cahn and CahnHilliard equations

4.1 Convex splitting schemes

4.2 Stabilized schemes

5 Numerical schemes for the case of matched density

5.1 Allen-Cahn phase-field model

5.2 Cahn-Hilliard phase-field model

6 Numerical schemes for the case of variable density and viscosity 
6.1 Allen-Cahn phase-field model

6.1.1 A scheme based on the Chorin-Temam projection method

6.1.2 Schemes based on pressure stabilization

\section{Introduction}

The interfacial dynamics of two-phase immiscible fluids have attracted much attention for more than a century. A classical way to study the moving interfaces is to employ a mesh that has grid points on the interfaces and deforms according to the motion of the boundary. However, keeping track of the moving mesh may cause computational difficulties such as mesh entanglement for large displacement or deformation of internal domains. Typically, sophisticated remeshing schemes have to be used in these cases. As an alternative, fixed-grid methods that regularize the interface have been highly successful in treating deforming interfaces. These include the volume-of-fluid method [43,44], the front-tracking method [24,25] and the level-set method [53,9]. Instead of formulating the flow in two domains separated by an interface, these methods represent the interfacial tension as a body-force or bulk-stress spreading over a narrow region covering the interface. A single set of governing equations can be written over the entire domain, and solved on a fixed grid in a purely Eulerian framework.

In recent years, the diffuse interface approach, i.e., the phase-field model, whose origin can be traced back to [61] and [71], has been successfully used to model two-phase incompressible fluids under various situations (cf. $[35,2,50,38,48,78]$ and the references therein). The phase field model can be viewed as a physically motivated level-set method. Instead of choosing an artificial smoothing function for the interface, the diffuse-interface model describes the interface by a mixing energy. In particular, the energetic variational phase-field approach leads to a well-posed nonlinear coupled system that satisfies an energy law, making it possible to design numerical schemes which satisfy a corresponding discrete energy law that automatically ensures their numerical stability (cf., for instance, [23, 45, 4, 63, 64]).

Most of the analysis and simulation of the phase-field model for twophase flows have been restricted to the matched density case or with a Boussinesq approximation when the density difference is small. The standard energetic phase-field model was derived from a variational procedure 
with a matched density, so a direct extension to the case of variable density does not lead to an energy law, making it difficult to carry out mathematical and numerical analysis. For problems with small density ratio, a common practice is to use a Boussinesq approximation where the variable density $\rho$ is replaced by a background constant density $\rho_{0}$ while an external gravitational force is added to model the effect of density difference (cf., for instance, [48]). While using the Boussinesq approximation leads to a wellposed dissipative phase-field system, its physical validity is based on the assumption that the density ratio between the two phases is small. Hence, it can not be used for two-phase flows with large density ratio. In this case, one approach is to view the mixture as a compressible fluid in the mixing layer with a mass-averaged velocity [50]. Another approach is to assume that the two components mix by advection only (i.e., without diffusion) and remain solenoidal. In [63], we took the second viewpoint and proposed a physically consistent modification to the momentum equation such that the modified phase-field system, in the case of variable density, admits an energy law.

The coupled phase-field system for two-phase incompressible flows presents formidable challenges for algorithm design, analysis and implementation, particularly so when the density ratio is large. Some of the numerical difficulties associated with this coupled nonlinear system include:

- the coupling of the velocity and pressure though the incompressibility constraint;

- the stiffness of the phase equation associated with the interfacial width $\eta$;

- and in the case of variable density, the equation to update the pressure becomes difficult to solve when the density ratio is large.

Some simple, efficient and energy stable schemes for the Allen-Cahn and Chan-Hilliard phase-field models have been constructed recently in [63] and [64]. These schemes are designed by combining the following approaches to deal with the aforementioned difficulties:

- A common strategy to decouple the computation of the pressure from velocity is to use a projection type scheme as in the case for Navier-Stokes equations (cf., for instance, a recent review in [32]). However, most of the numerical analysis for projection type schemes are limited to problems with constant density and viscosity. The few exceptions which deal with variable density are 
$[28,56,29]$, which serve as inspiration for the numerical schemes we present below.

- To alleviate the difficulty associated with the stiffness caused by the thin interfacial width, a stabilizing term in the phase equation was introduced in $[77,46]$ (see also [75]). This stabilizing term allows us to treat the nonlinear term in the phase equation explicitly without suffering from any time step constraint. Alternatively, one can also use the so called convex splitting approach [21] which leads to unconditionally stable scheme with a nonlinear system whose solution is the unique minimizer of a convex functional (cf. [74,73]).

- A typical projection type scheme will lead to an elliptic equation with density as a variable coefficient for the pressure. The numerical solution of this elliptic equation could become very expensive as the when the density ratio is large. We shall consider a pressure stabilized formulation to avoid solving a pressure elliptic equation with density as a variable coefficient (cf. [29]).

The main purpose of this note is to present a unified approach for the design and stability analysis of simple, efficient and energy stable schemes for the Allen-Cahn and Cahn-Hilliard phase-field models. While many of the schemes and results to be discussed here have appeared in several recent papers $[65,63,64]$, the presentation is essentially self-contained and in a uniform style.

The rest of this note is organized as follows. In the next section, we present the Allen-Cahn and Cahn-Hilliard phase-field models for two-phase incompressible Newtonian flows, first with the case matched density afollowed by the case of variable density. We emphasize that these phase-field models admit an energy law. In Section 3, we provide a brief overview for some projection type schemes for the Navier-Stokes equations that we shall use to decouple the computation of the pressure from that of the velocity. In Section 4, we present two classes of energy stable schemes for the Allen-Cahn and Cahn-Hilliard equations. In Section 5, we consider the phase-field models with matched density and viscosity, and construct energy stable schemes by combining the ideas presented in Sections 3 and 4. In Section 6, we extend the results to the case of variable density and viscosity. We discuss briefly the extension to complex fluids in Section 7 and conclude with a few remarks in the last section. 


\section{2. phase-field model for two-phase incompressible and immiscible flows}

We consider a mixture of two immiscible, incompressible Newtonian fluids with densities $\rho_{1}, \rho_{2}$ and viscosities $\mu_{1}, \mu_{2}$. In order to identify the regions occupied by the two fluids, we introduce a phase function $\phi$ such that

$$
\phi(x, t)=\left\{\begin{array}{ll}
1 & \text { fluid } 1 \\
-1 & \text { fluid } 2
\end{array},\right.
$$

with a thin smooth transition layer of thickness $\eta$ connecting the two fluids so the interface of the mixture can be described by the zero level set of the phase function: $\{x: \phi(x, t)=0\}$. Let $F(\phi)=\frac{1}{4 \eta^{2}}\left(\phi^{2}-1\right)^{2}$ be the GinzburgLandau double-well potential, and define the mixing energy functional

$$
W(\phi, \nabla \phi)=\lambda \int_{\Omega}\left(\frac{1}{2}|\nabla \phi|^{2}+F(\phi)\right) d x,
$$

where $\lambda$ is the mixing energy density and $\eta$ is the capillary width of the interface thickness. The above represents the competition between the hydrophilic and hydro-phobic properties of the two-phase flow. The mixing energy density $\lambda$ can be related to the traditional surface tension coefficient $\sigma$ in the sharp interface limit [78]. Indeed, consider an one-dimensional interface and assume that the diffusive mixing energy in the region equals to the traditional surface energy:

$$
\sigma=\lambda \int_{-\infty}^{+\infty}\left\{\frac{1}{2}\left(\frac{d \phi}{d x}\right)^{2}+F(\phi)\right\} d x
$$

Let us further assume that the diffuse interface is at equilibrium, and thus has zero chemical potential,

$$
\frac{\delta W}{\delta \phi}=\lambda\left\{-\frac{d^{2} \phi}{d x^{2}}+F^{\prime}(\phi)\right\}=0 .
$$

Since $\lim _{x \rightarrow \pm \infty} F(\phi)=0$, that is, $\phi= \pm 1$ for unmixed components, and $\lim _{x \rightarrow \pm \infty} \frac{d \phi}{d x}=0$, multiplying the above equation by $\frac{d \phi}{d x}$ and integrating over $(-\infty,+\infty)$, we get

$$
\frac{1}{2}\left(\frac{d \phi}{d x}\right)^{2}=F(\phi)
$$

which implies equal partition of the free energy between the two terms at equilibrium. Equation (2.5) can be solved together with the boundary condition $\phi(0)=0$ for any given $F(\phi)$ by direct integration. For the potential 
$F(\phi)=\frac{1}{4 \eta^{2}}\left(1-\phi^{2}\right)^{2},(2.5)$ leads to $\phi_{x}=\frac{1}{\sqrt{2} \eta}\left(1-\phi^{2}\right)$ whose solution, with the condition $\phi(0)=0$, is:

$$
\phi(x)=\tanh \left(\frac{x}{\sqrt{2} \eta}\right) .
$$

Thus, at the equilibrium, the capillary width $\eta$ is a measure of the thickness of the diffuse interface. Substituting (2.6) into (2.3), we arrive at the following matching condition:

$$
\sigma=\frac{2 \sqrt{2}}{3} \frac{\lambda}{\eta}
$$

Therefore, as the interfacial thickness $\eta$ shrinks toward zero, so should the mixing energy density parameter $\lambda$; their ratio gives the surface tension coefficient in the sharp interface limit.

Obviously, the correspondence between the diffuse- and sharp-interface models is meaningful only when the former is at equilibrium. During the relaxation of the diffuse interface, one cannot speak of a constant interfacial tension. Although one may view this as a deficiency of the diffuse-interface model, it in fact reflects the reality that the interface has its own dynamics which cannot be summarized by a constant $\sigma$ except under limiting conditions.

The dynamics of the phase function $\phi$ can be determined by a gradient flow:

$$
\phi_{t}+(u \cdot \nabla) \phi=-\gamma \frac{\delta W}{\delta \phi} .
$$

One can take the variational derivative $\frac{\delta W}{\delta \phi}$ in $H^{-1}$ and set $f(\phi)=F^{\prime}(\phi)$, leading to the (conserved) Cahn-Hilliard phase equation,

$$
\phi_{t}+(u \cdot \nabla) \phi=-\gamma \Delta(\Delta \phi-f(\phi)) ;
$$

or take the variational derivative $\frac{\delta W}{\delta \phi}$ in $L^{2}$, leading to the (non-conserved) Allen-Cahn phase equation

$$
\phi_{t}+(u \cdot \nabla) \phi=\gamma(\Delta \phi-f(\phi)) .
$$

For immiscible two-phase flows, we can introduce a non-local Lagrange multiplier $\xi(t)$ in the Allen-Cahn case to enforce the volume fraction conservation (cf. [77]), namely:

$$
\begin{aligned}
& \phi_{t}+(u \cdot \nabla) \phi=\gamma(\Delta \phi-f(\phi)+\xi(t)), \\
& \frac{d}{d t} \int_{\Omega} \phi d x=0 .
\end{aligned}
$$


Note that the right-hand side of the dynamic equation (2.9) or (2.10) dictates the relaxation of the phase-field variable $\phi$, with a relaxation time proportional to $1 / \gamma$. In the limit of $\gamma$ approaching zero, we recover the kinematic condition for the interface. Moreover, as $\eta$ approaches zero, the dynamics of $\phi$ will preserve the profile of the transition, a key advantage of the phase-field approach.

The momentum equation for the two-phase system takes the usual form:

$$
\rho\left(u_{t}+(u \cdot \nabla) u\right)=\nabla \cdot \tau,
$$

where the total stress $\tau=\mu D(u)-p I+\tau_{e}$ with $\mu$ being the dynamic viscosity coefficient, $D(u)=\nabla u+\nabla u^{T}$ and that $\tau_{e}$ is the extra elastic stress induced by the interfacial surface tension.

\subsection{The case of matched density}

We consider first the case $\rho_{1}=\rho_{2}=\rho_{0}$. Then, using the least-actionprinciple and the mixing energy functional defined above, it can be shown that $\tau_{e}=-\lambda \nabla \phi \otimes \nabla \phi$ (cf. $\left.[34,48,57]\right)$. Using the identity

$$
\nabla \cdot(\nabla \phi \otimes \nabla \phi)=\Delta \phi \nabla \phi+\frac{1}{2} \nabla|\nabla \phi|^{2},
$$

and absorbing the gradient term into the pressure $p$, the momentum equation becomes:

$$
\begin{aligned}
\rho_{0}\left(u_{t}+(u \cdot \nabla) u\right) & =\nabla \cdot(\mu D(u)-p I-\lambda(\nabla \phi \otimes \nabla \phi)) \\
& =\nabla \cdot(\mu D(u))-\nabla p-\lambda \Delta \phi \nabla \phi,
\end{aligned}
$$

where, for notational simplicity, we still use $p$ to denote the modified pressure $p+\frac{\lambda}{2}|\nabla \phi|^{2}$. Note that $\mu$ is a slave variable of $\phi$ and can be determined, for example, by

$$
\mu(\phi)=\frac{\mu_{1}-\mu_{2}}{2} \phi+\frac{\mu_{1}+\mu_{2}}{2} .
$$

Then, the Allen-Cahn phase field model for the case of matched density and viscosity consists of (2.11), (2.14) and the incompressibility condition

$$
\nabla \cdot u=0
$$

together with the boundary conditions

$$
\left.u\right|_{\partial \Omega}=0,\left.\quad \frac{\partial \phi}{\partial n}\right|_{\partial \Omega}=0,
$$

and initial conditions

$$
\left.u\right|_{t=0}=u_{0}, \quad \phi_{t=0}=\phi_{0} .
$$


By taking the inner product of (2.14) with $u$, and (2.11) with $-\lambda(\Delta \phi-$ $f(\phi)+\xi(t))$, we can derive the following energy law:

$$
\begin{aligned}
\frac{d}{d t}\left\{\frac{\rho_{0}}{2}\|u\|^{2}\right. & \left.+\frac{\lambda}{2}\|\nabla \phi\|^{2}+\lambda(F(\phi), 1)\right\} \\
& =-\frac{1}{2}\|\sqrt{\mu} D(u)\|^{2}-\lambda \gamma\|\Delta \phi-f(\phi)+\xi(t)\|^{2} .
\end{aligned}
$$

On the other hand, the Cahn-Hilliard phase-field model consists of (2.9), (2.14) and (2.16) together with the boundary conditions

$$
\left.u\right|_{\partial \Omega}=0,\left.\quad \frac{\partial \phi}{\partial n}\right|_{\partial \Omega}=0,\left.\quad \frac{\partial(\Delta \phi-f(\phi))}{\partial n}\right|_{\partial \Omega}=0,
$$

and the initial conditions (2.18). Similarly, by taking the inner product of (2.14) with $u$, and (2.9) with $-\lambda(\Delta \phi-f(\phi)+\xi(t))$, we derive the following energy law for the Cahn-Hilliard phase-field model:

$$
\begin{aligned}
\frac{d}{d t}\left\{\frac{\rho_{0}}{2}\|u\|^{2}\right. & \left.+\frac{\lambda}{2}\|\nabla \phi\|^{2}+\lambda(F(\phi), 1)\right\} \\
& =-\frac{1}{2}\|\sqrt{\mu} D(u)\|^{2}-\lambda \gamma\|\nabla(\Delta \phi-f(\phi))\|^{2} .
\end{aligned}
$$

\subsection{The case of variable density}

When the density ratio is small, a usual approach is to use the Boussinesq approximation, i.e., replacing (2.14) by

$$
\rho_{0}\left(u_{t}+(u \cdot \nabla) u\right)=\nabla \cdot(\mu D(u))-\nabla p-\lambda \Delta \phi \nabla \phi+g\left(\rho_{1}, \rho_{2}\right),
$$

where $\rho_{0}=\frac{\rho_{1}+\rho_{2}}{2}$ and $g\left(\rho_{1}, \rho_{2}\right)$ is an additional gravitational force to account for the density difference. However, when the density ratio is large, the above Boussinesq approximation is no longer valid. It is clear that the momentum equation should now be

$$
\rho\left(u_{t}+(u \cdot \nabla) u\right)=\nabla \cdot(\mu D(u))-\nabla p-\lambda \Delta \phi \nabla \phi .
$$

However, unlike in the matched density case, the mass conservation (which was not included in the phase-field system in favor of the phase equation)

$$
\rho_{t}+\nabla \cdot(\rho u)=0,
$$

is no longer a consequence of incompressibility (2.16). Therefore, the corresponding Allen-Cahn (resp. Cahn-Hilliard) phase-field model (2.11) (resp. $(2.9)),(2.23)$ with the incompressibility condition (2.16) no longer admit an energy law. In this case, one approach is to view the mixture as a compressible fluid with $\nabla \cdot u \neq 0$ in the mixing layer, where $u$ is a mass-averaged 
velocity [50]. Another approach [63] is to assume the two components mix by advection only (i.e., without diffusion). Thus, the velocity at a spatial point is defined as that of the component occupying that point; it is spatially continuous and remains solenoidal. We then need to modify $(2.23)$ in a physically consistent way for the modified system to have an energy law. To this end, we set $\sigma=\sqrt{\rho}$. By using the mass conservation (2.24), we have

$$
\sigma(\sigma u)_{t}=\rho u_{t}+\frac{1}{2} \rho_{t} u=\rho u_{t}-\frac{1}{2} \nabla \cdot(\rho u) u .
$$

Therefore, it is physically consistent to replace $\rho u_{t}$ in $(2.23)$ by $\sigma(\sigma u)_{t}+$ $\frac{1}{2} \nabla \cdot(\rho u) u$, leading to the modified momentum equation [63]

$$
\begin{aligned}
\sigma(\sigma u)_{t}+(\rho u \cdot \nabla) u & +\frac{1}{2} \nabla \cdot(\rho u) u \\
& -\nabla \cdot \mu D(u)+\nabla p+\lambda \Delta \phi \nabla \phi=0 .
\end{aligned}
$$

In the above $\rho$ and $\mu$ are slave variables of $\phi$, given by

$$
\rho(\phi)=\frac{\rho_{1}-\rho_{2}}{2} \phi+\frac{\rho_{1}+\rho_{2}}{2}, \mu(\phi)=\frac{\mu_{1}-\mu_{2}}{2} \phi+\frac{\mu_{1}+\mu_{2}}{2} .
$$

Thanks to the property that

$$
\int_{\Omega}(\rho u \cdot \nabla) v \cdot v d x+\frac{1}{2} \int_{\Omega} \nabla \cdot(\rho u) v \cdot v d x=0, \text { if }\left.u \cdot n\right|_{\partial \Omega}=0,
$$

we can then derive that the Allen-Cahn phase-field model for the case of variable density (2.11), (2.26), (2.16) with (2.17) satisfies the following energy law:

$$
\begin{aligned}
\frac{d}{d t}\left\{\frac{1}{2}\|\sqrt{\sigma} u\|^{2}\right. & \left.+\frac{\lambda}{2}\|\nabla \phi\|^{2}+\lambda(F(\phi), 1)\right\} \\
& =-\frac{1}{2}\|\sqrt{\mu} D(u)\|^{2}-\lambda \gamma\|\Delta \phi-f(\phi)+\xi(t)\|^{2} .
\end{aligned}
$$

Similarly, for the the Cahn-Hilliard phase-field model for the case of variable density (2.9), (2.26), (2.16) with (2.20), we have

$$
\begin{aligned}
\frac{d}{d t}\left\{\frac{1}{2}\|\sqrt{\sigma} u\|^{2}\right. & \left.+\frac{\lambda}{2}\|\nabla \phi\|^{2}+\lambda(F(\phi), 1)\right\} \\
& =-\frac{1}{2}\|\sqrt{\mu} D(u)\|^{2}-\lambda \gamma\|\nabla(\Delta \phi-f(\phi))\|^{2} .
\end{aligned}
$$

It is a great challenge to design simple and efficient schemes for solving the coupled, nonlinear Allen-Cahn and Cahn-Hilliard phase-field systems described above. In the next two sections, we shall describe simple and efficient schemes for solving the Navier-Stokes equations and AllenCahn/Cahn-Hilliard equations, respectively. Then, we shall use the ideas 
in the next two sections as building blocks to design simple, efficient and energy stable schemes for the Allen-Cahn and Cahn-Hilliard phase-field systems in Sections 7 and 8.

\section{Some efficient time discretization schemes for Navier-Stokes equations}

We consider in this section the time dependent Navier-Stokes equations:

$$
\begin{aligned}
& u_{t}+(u \cdot \nabla) u=\nu \Delta u-\nabla p+f, \quad(x, t) \in \Omega \times(0, T], \\
& \nabla \cdot u=0, \quad(x, t) \in \Omega \times(0, T], \\
& \left.u\right|_{\partial \Omega=0},\left.\quad u\right|_{t=0}=u_{0} .
\end{aligned}
$$

In the above, $\Omega \subset R^{d}(d=2,3)$ is a bounded domain; $\nu$ is the kinematic viscosity coefficient, $f$ is the external force; the unknown functions are the velocity vector $u$ and the pressure $p$.

To fix the idea, we assumed a homogeneous Dirichlet boundary condition in the above, although other admissible boundary conditions can be treated as well.

One of the main difficulties in numerically solving the Navier-Stokes equations is that the pressure $p$ is coupled with the velocity $u$ through the incompressibility constraint for $u$. The most effective way to decouple the computation of pressure from that of the velocity is to employ a projection type scheme which was originally proposed by Chorin [12] and Temam [69]. Many improved versions have been introduced over the last forty years (cf., for instance, [26, 41, 72, 52, 40, 66, 54, 67, 70, 20, 30, 31, 33, 39]); and an extensive literature has been devoted to the numerical analysis of these projection type schemes (cf., for instance, $[59,66,36,18,19,67,55,27,58,6,30,31,33,51,49])$. We refer to the recent review paper [32] for a detailed account on the various type of projection schemes. Here, we shall describe two projection type schemes which will be used later for the phase-phase model.

\subsection{Pressure-correction schemes}

The original projection method, proposed by Chorin [12] and Temam [69], was motivated by the idea of operator splitting, its semi-discrete version reads as follows: Find $\tilde{u}^{k+1}$ by solving

$$
\begin{aligned}
& \frac{\tilde{u}^{k+1}-u^{k}}{\delta t}-\nu \Delta \tilde{u}^{k+1}+\left(u^{k} \cdot \nabla\right) \tilde{u}^{k+1}=f\left(t_{k+1}\right), \\
& \left.\tilde{u}^{k+1}\right|_{\partial \Omega}=0
\end{aligned}
$$


then, find $\left(p^{k+1}, u^{k+1}\right)$ by solving

$$
\begin{aligned}
& \frac{u^{k+1}-\tilde{u}^{k+1}}{\delta t}+\nabla p^{k+1}=0, \\
& \nabla \cdot u^{k+1}=0 \\
& \left.u^{k+1} \cdot n\right|_{\partial \Omega}=0 .
\end{aligned}
$$

The above scheme is called a projection method because $u^{n+1}$ in the second step (3.3) can be interpreted as the projection of $\tilde{u}^{n+1}$ onto the divergence free space

$$
H=\left\{u \in\left(L^{2}(\Omega)\right)^{d}: \nabla \cdot u=0,\left.u \cdot n\right|_{\partial \Omega}=0\right\} .
$$

By taking the divergence of the first equation in (3.3), we find that the second step (3.3) is equivalent to

$$
\begin{aligned}
& \Delta p^{k+1}=\frac{1}{\delta t} \nabla \cdot \tilde{u}^{k+1},\left.\quad \frac{\partial p^{k+1}}{\partial n}\right|_{\partial \Omega}=0 \\
& u^{k+1}=\tilde{u}^{k+1}-\delta t \nabla p^{k+1} .
\end{aligned}
$$

Remark 3.1: The above scheme is computationally efficient, when compared with a coupled discretization, since at each time step, one only needs to solve a linear second-order equation for $\tilde{u}^{k+1}$, and a Poisson equation for $p^{k+1}$. Furthermore, it is a common practice, especially when a fast Poisson solver is available as in the case of finite differences or spectral methods, to replace the semi-implicit treatment of the nonlinear term $\left(u^{k} \cdot \nabla\right) \tilde{u}^{k+1}$ in (3.2) by the explicit treatment $\left(u^{k} \cdot \nabla\right) \tilde{u}^{k}$. Therefore, at each time step, one only needs to solve a Poisson type equation for $\tilde{u}^{k+1}$ and a Poisson equation for $p^{k+1}$.

On the other hand, this scheme has a poor accuracy due to the nonphysical boundary condition $\left.\frac{\partial p^{k+1}}{\partial n}\right|_{\partial \Omega}=0$ in (3.5). It is shown in [66] that this scheme is only $\frac{1}{2}$-order accurate for the velocity in $L^{2}\left(0, T ; H^{1}\right)$ and pressure in $L^{2}\left(0, T ; L^{2}\right)$, and first-order accurate for the velocity in $L^{2}\left(0, T ; L^{2}\right)$.

The first significantly improved projection type scheme appears to be the so called pressure-correction scheme introduced in [26]. Its first-order version reads as follows:

Find $\tilde{u}^{k+1}$ by solving

$$
\begin{aligned}
& \frac{\tilde{u}^{k+1}-u^{k}}{\delta t}-\nu \Delta \tilde{u}^{k+1}+\left(u^{k} \cdot \nabla\right) \tilde{u}^{k+1}+\nabla p^{k}=f\left(t_{k+1}\right), \\
& \left.\tilde{u}^{k+1}\right|_{\partial \Omega}=0
\end{aligned}
$$


then, find $\left(p^{k+1}, u^{k+1}\right)$ by solving

$$
\begin{aligned}
& \frac{u^{k+1}-\tilde{u}^{k+1}}{\delta t}+\nabla\left(p^{k+1}-p^{k}\right)=0, \\
& \nabla \cdot u^{k+1}=0, \\
& \left.u^{k+1} \cdot n\right|_{\partial \Omega}=0 .
\end{aligned}
$$

By taking the divergence of the first equation in (3.7), we find that the second step is equivalent to

$$
\begin{aligned}
& \Delta\left(p^{k+1}-p^{k}\right)=\frac{1}{\delta t} \nabla \cdot \tilde{u}^{k+1},\left.\quad \frac{\partial\left(p^{k+1}-p^{k}\right)}{\partial n}\right|_{\partial \Omega}=0 \\
& u^{k+1}=\tilde{u}^{k+1}-\delta t \nabla\left(p^{k+1}-p^{k}\right) .
\end{aligned}
$$

Taking the inner product of (3.6) with $2 \delta t \tilde{u}^{k+1}$, using the identity

$$
(b-a, 2 b)=|b|^{2}-|a|^{2}+|b-a|^{2}
$$

and the property

$$
(u \cdot \nabla v, v)=0, \quad \forall u \in H, v \in\left(H^{1}(\Omega)\right)^{d},
$$

where $H$ is defined in (3.4), we find

$$
\begin{aligned}
\left\|\tilde{u}^{k+1}\right\|^{2} & -\left\|\tilde{u}^{k}\right\|^{2}+\left\|\tilde{u}^{k+1}-u^{k}\right\|^{2}+2 \nu \delta t\left\|\nabla \tilde{u}^{k+1}\right\|^{2}+2 \delta t\left(\nabla p^{k}, \tilde{u}^{k+1}\right) \\
& =2 \delta t\left(f\left(t_{k+1}\right), \tilde{u}^{k+1}\right) \leq \nu \delta t\left\|\nabla \tilde{u}^{k+1}\right\|^{2}+C \delta t\left\|f\left(t_{k+1}\right)\right\|_{H^{-1}}^{2} .
\end{aligned}
$$

On the other hand, we rewrite (3.7) as

$$
u^{k+1}+\delta t \nabla p^{k+1}=\tilde{u}^{k+1}+\delta t \nabla p^{k}
$$

and take the inner product of the above with itself on both sides, notice that $\left(\nabla p^{k+1}, u^{k+1}\right)=-\left(p^{k+1}, \nabla \cdot u^{k+1}\right)=0$, we obtain

$$
\left\|u^{k+1}\right\|^{2}+(\delta t)^{2}\left\|\nabla p^{k+1}\right\|^{2}=\left\|\tilde{u}^{k+1}\right\|^{2}+2 \delta t\left(\nabla p^{k}, \tilde{u}^{k+1}\right)+(\delta t)^{2}\left\|\nabla p^{k}\right\|^{2} .
$$

Summing up (3.11) and (3.12), we arrive at

$$
\begin{aligned}
\left\|u^{k+1}\right\|^{2}-\left\|\tilde{u}^{k}\right\|^{2} & +\left\|\tilde{u}^{k+1}-u^{k}\right\|^{2}+\nu \delta t\left\|\nabla \tilde{u}^{k+1}\right\|^{2} \\
& +(\delta t)^{2}\left\|\nabla p^{k+1}\right\|^{2}-(\delta t)^{2}\left\|\nabla p^{k}\right\|^{2} \leq C \delta t\left\|f\left(t_{k+1}\right)\right\|_{H^{-1}}^{2} .
\end{aligned}
$$

Summing up the above inequality for $k=0,1, \ldots, n \leq \frac{T}{\delta t}$, we proved the following result. 
Lemma 3.1: The scheme (3.6)-(3.7) is unconditionally stable in the sense that

$$
\left\|u^{n+1}\right\|^{2}+\nu \delta t \sum_{k=0}^{n}\left\|\nabla \tilde{u}^{k+1}\right\|^{2} \leq\left\|u^{0}\right\|^{2}+C\|f\|_{C\left(0, T ; H^{-1}\right)}^{2} .
$$

It is also shown that the above scheme is first-order accurate for the velocity in $L^{\infty}\left(0, T ; L^{2}\right) \cap L^{2}\left(0, T ; H^{1}\right)$ and pressure in $L^{2}\left(0, T ; L^{2}\right)$ (cf. $[66,32])$. However, second-order pressure-correction schemes can be easily constructed. A popular second-order version reads:

$$
\begin{aligned}
& \frac{3 \tilde{u}^{k+1}-4 u^{k}+u^{k-1}}{2 \delta t}-\nu \Delta \tilde{u}^{k+1}+\left(2 u^{k}-u^{k-1}\right) \cdot \nabla \tilde{u}^{k+1}+\nabla p^{k}=f\left(t_{k+1}\right), \\
& \left.\tilde{u}^{k+1}\right|_{\partial \Omega}=0
\end{aligned}
$$

$$
\begin{aligned}
& \frac{3 u^{k+1}-3 \tilde{u}^{k+1}}{2 \delta t}+\nabla\left(p^{k+1}-p^{k}\right)=0, \\
& \nabla \cdot u^{k+1}=0, \\
& \left.u^{k+1} \cdot n\right|_{\partial \Omega}=0 .
\end{aligned}
$$

It can be shown, similar to the proof for Lemma 3.1, that the above scheme is also unconditionally stable. The only complication is that the term $\left(3 \tilde{u}^{k+1}-4 u^{k}+u^{k-1}, \tilde{u}^{k+1}\right)$ needs to be treated carefully, we refer to $[27,33]$ for more detail on this matter. As for the error analysis, it is shown (cf. $[27,68])$ that the above scheme is second-order for the velocity in the $L^{2}\left(0, T ; L^{2}(\Omega)\right)$-norm, but it is only first-order accurate for the velocity in $L^{2}\left(0, T ; H^{1}(\Omega)\right)$-norm and the pressure in $L^{2}\left(0, T ; L^{2}(\Omega)\right)$-norm. The proof is much more involved and we refer to $[27,32]$ for more detail.

The scheme (3.13)-(3.14) is only first-order accurate for the pressure [68] due to the non-physical boundary condition

$$
\left.\frac{\partial p^{k+1}}{\partial n}\right|_{\partial \Omega}=\left.\frac{\partial p^{k}}{\partial n}\right|_{\partial \Omega}=\cdots=\left.\frac{\partial p^{0}}{\partial n}\right|_{\partial \Omega}
$$

implied by (3.14). An improved second-order scheme, is proposed in [70]:

$$
\begin{aligned}
& \frac{3 \tilde{u}^{k+1}-4 u^{k}+u^{k-1}}{2 \delta t}-\nu \Delta \tilde{u}^{k+1}+\left(2 u^{k}-u^{k-1}\right) \cdot \nabla \tilde{u}^{k+1}+\nabla p^{k}=f\left(t_{k+1}\right), \\
& \left.\tilde{u}^{k+1}\right|_{\partial \Omega}=0
\end{aligned}
$$




$$
\begin{aligned}
& \frac{3 u^{k+1}-3 \tilde{u}^{k+1}}{2 \delta t}+\nabla \psi^{k+1}=0, \\
& \nabla \cdot u^{k+1}=0, \\
& \left.u^{k+1} \cdot n\right|_{\partial \Omega}=0 ; \\
& p^{k+1}=\psi^{k+1}+p^{k}-\frac{2 \nu \delta t}{3} \nabla \cdot \tilde{u}^{k+1} .
\end{aligned}
$$

It is shown (cf. [33]) that the above scheme is still unconditionally stable but its accuracy is improved to order $\frac{3}{2}$, instead of first-order for the scheme (3.13)-(3.14), for the velocity in $L^{2}\left(0, T ; H^{1}(\Omega)\right)$-norm and the pressure in $L^{2}\left(0, T ; L^{2}(\Omega)\right)$-norm.

\subsection{Pressure-stabilization schemes}

As we shall see later, for two-phase flows with variable density, the pressurecorrection schemes presented above will lead to a pressure elliptic equation with variable coefficients which may become difficult to solve when the density ratio is large. Hence, we shall present below a pressure-stabilization scheme which will lead to a pressure Poisson equation even in the case of variable density.

The idea behind the pressure-stabilization scheme is to replace the divergence free condition by

$$
\nabla \cdot u-\epsilon \Delta p=0,\left.\quad \frac{\partial p}{\partial n}\right|_{\partial \Omega}=0
$$

with $\epsilon=\delta t$ or

$$
\nabla \cdot u-\epsilon \Delta p_{t}=0,\left.\quad \frac{\partial p_{t}}{\partial n}\right|_{\partial \Omega}=0
$$

with $\epsilon=(\delta t)^{2}$.

A first-order pressure-stabilization scheme based on (3.19) reads:

$$
\begin{gathered}
\frac{u^{k+1}-u^{k}}{\delta t}-\nu \Delta \tilde{u}^{k+1}+N\left(u^{k}, u^{k+1}\right)+\nabla p^{k}=f\left(t_{k+1}\right) \\
\left.\tilde{u}^{k+1}\right|_{\partial \Omega}=0 \\
\delta t \Delta p^{k+1}=\nabla \cdot u^{k+1},\left.\quad \frac{\partial p^{k+1}}{\partial n}\right|_{\partial \Omega}=0
\end{gathered}
$$

where

$$
N\left(u^{k}, u^{k+1}\right)=\left(u^{k} \cdot \nabla\right) u^{k+1}+\frac{1}{2}\left(\nabla \cdot u^{k}\right) u^{k+1} .
$$


Note that in the above scheme, $\nabla \cdot u^{k} \neq 0$. Hence, we don't have the desired property $\left(\left(u^{k} \cdot \nabla\right) u^{k+1}, u^{k+1}\right)=0$. By adding an additional term $\frac{1}{2}(\nabla$. $\left.u^{k}\right) u^{k+1}$, we have $\left(N\left(u^{k}, u^{k+1}\right), u^{k+1}\right)=0$ which is essential for proving the stability of the scheme.

It is shown in [59] that the original projection method introduced in [12] and [69] can be reformulated as the stabilized scheme (3.21)-(3.22). Therefore, Its accuracy is comparable to the original projection method but not as accurate as the first-order scheme (3.6)-(3.7). Namely, it is of first order for the velocity in the $L^{2}\left(0, T ; L^{2}(\Omega)\right)$-norm, but it is only of order $\frac{1}{2}$ for the velocity in $L^{2}\left(0, T ; H^{1}(\Omega)\right)$-norm and the pressure in $L^{2}\left(0, T ; L^{2}(\Omega)\right)$-norm (cf. [59]).

The computational complexity of the scheme (3.21)-(3.22) is essentially the same as that of the scheme (3.6)-(3.7). However, as we shall see later, it leads to a simplified scheme for two-phase flows with variable density.

A second-order pressure-stabilization scheme based on (3.20) is as follows:

$$
\begin{aligned}
& \frac{3 u^{k+1}-4 u^{k}+u^{k-1}}{2 \delta t}-\nu \Delta \tilde{u}^{k+1}+N\left(2 u^{k}-u^{k-1}, u^{k+1}\right)+\nabla p^{k}=f\left(t_{k+1}\right), \\
& \left.\tilde{u}^{k+1}\right|_{\partial \Omega}=0
\end{aligned}
$$

$$
\delta t \Delta\left(p^{k+1}-p^{k}\right)=\nabla \cdot u^{k+1},\left.\quad \frac{\partial p^{k+1}}{\partial n}\right|_{\partial \Omega}=0 .
$$

The accuracy of the second-order scheme (3.24)-(3.25) is essentially the same as the second-order pressure-correction scheme (3.13)-(3.14) (cf. [67]).

Remark 3.2: For all the pressure-correction schemes, the pressure computation is decoupled from that of the velocity. Therefore, it appears that the discretization spaces, $X_{h}$ for the velocity and $M_{h}$ for the pressure, do not necessarily need to satisfy the so called inf-sup condition [3,5]. However, it has been shown that the inf-sup condition is needed for the pressurecorrection schemes to achieve optimal accuracy (see, for instance, a detailed explanation in [32]). 


\section{Some efficient time discretization schemes for Allen-Cahn and Cahn-Hilliard equations}

We consider in this section the Allen-Cahn equation

$$
\begin{aligned}
& \phi_{t}-\Delta \phi+\frac{1}{\varepsilon^{2}} f(\phi)=0, \quad(x, t) \in \Omega \times(0, T], \\
& \left.\frac{\partial}{\partial n} \phi\right|_{\partial \Omega}=0
\end{aligned}
$$

and the Cahn-Hilliard equation

$$
\begin{aligned}
& \phi_{t}-\Delta\left(-\Delta \phi+\frac{1}{\varepsilon^{2}} f(\phi)\right)=0, \quad(x, t) \in \Omega \times(0, T], \\
& \left.\frac{\partial}{\partial n} \phi\right|_{\partial \Omega}=0,\left.\quad \frac{\partial}{\partial n}\left(\Delta \phi-\frac{1}{\varepsilon^{2}} f(\phi)\right)\right|_{\partial \Omega}=0,
\end{aligned}
$$

with the initial condition $\left.\phi\right|_{t=0}=\phi_{0}$. In the above, $f(\phi)=F^{\prime}(\phi)$ with $F(\phi)$ being a given energy potential, and $n$ is the outward normal. In many situations, it is more convenient to rewrite the fourth-order equation (4.2) into a system of second-order equations:

$$
\begin{aligned}
\phi_{t} & =\Delta w,\left.\quad \frac{\partial}{\partial n} w\right|_{\partial \Omega}=0, \\
w & =-\Delta \phi+\frac{1}{\varepsilon^{2}} f(\phi),\left.\quad \frac{\partial}{\partial n} \phi\right|_{\partial \Omega}=0 .
\end{aligned}
$$

We have introduced a parameter $\varepsilon$ explicitly in (4.1) and (4.2) in order to highlight additional difficulties which may arise when $\varepsilon \ll 1$.

The Allen-Cahn equation was originally introduced by Allen and Cahn in [1] to describe the motion of anti-phase boundaries in crystalline solids, while the Cahn-Hilliard equation was introduced by Cahn and Hilliard in [8] to describe the complicated phase separation and coarsening phenomena in a solid. These equations have been used extensively in materials science as models for phase transition, pattern formation etc., and have been the subject of extensive mathematical and numerical studies. On the other hand, the Allen-Cahn and Cahn-Hilliard equations have also been widely in phase-field models for multi-phase flows (cf., for instance, $[50,11,2,10,48,78,77,16])$.

An important feature of the Allen-Cahn and Cahn-Hilliard equations is that they can be viewed as the gradient flow of the Liapunov energy functional

$$
E(\phi):=\int_{\Omega}\left(\frac{1}{2}|\nabla \phi|^{2}+\frac{1}{\varepsilon^{2}} F(\phi)\right) d x
$$


in $L^{2}$ and $H^{-1}$, respectively. More precisely, by taking the inner product of (4.1) with $-\Delta \phi+\frac{1}{\varepsilon^{2}} f(\phi)$, we immediately find the following energy law for $(4.1)$ :

$$
\frac{\partial}{\partial t} E(\phi(t))=-\int_{\Omega}\left|-\Delta \phi+\frac{1}{\varepsilon^{2}} f(\phi)\right|^{2} d x ;
$$

and similarly, the energy law for (4.2) is

$$
\frac{\partial}{\partial t} E(\phi(t))=-\int_{\Omega}\left|\nabla\left(-\Delta \phi+\frac{1}{\varepsilon^{2}} f(\phi)\right)\right|^{2} d x .
$$

Therefore, it is important to design efficient and accurate numerical schemes that satisfy a corresponding discrete energy law, or in other words, energy stable.

Let us consider first a simple first-order semi-implicit scheme for the Allen-Cahn equation (4.1):

$$
\frac{1}{\delta t}\left(\phi^{n+1}-\phi^{n}\right)=\Delta \phi^{n+1}-\frac{1}{\varepsilon^{2}} f\left(\phi^{n}\right),\left.\quad \frac{\partial}{\partial n} \phi^{n+1}\right|_{\partial \Omega}=0 .
$$

In order to prove a stability result for the above scheme, we need to make the following assumption on $f(\phi)=F^{\prime}(\phi)$ :

$$
\max _{x \in \mathbb{R}}\left|f^{\prime}(x)\right| \leq L
$$

Note that the assumption (4.8) is not satisfied by the double-well potential $F(\phi)=\frac{1}{4}\left(\phi^{2}-1\right)^{2}$. However, since the Allen-Cahn equation satisfies the maximum principle and it is shown in [7] that for a truncated potential $F(\phi)$ with quadratic growth at infinities, the maximum norm of the solution for the Cahn-Hilliard equation is bounded, we can replace $F(\phi)$ by

$$
F(\phi)= \begin{cases}(\phi-1)^{2}, & \phi>1 \\ \frac{1}{4}\left(\phi^{2}-1\right)^{2}, & \phi \in[-1,1] \\ (\phi+1)^{2}, & \phi<-1,\end{cases}
$$

which satisfies (4.8) with $L=2$.

Under the assumption (4.8), it is not hard to show that under the condition

$$
\delta t \leq \frac{2 \varepsilon^{2}}{L}
$$

the scheme (4.7) is energy stable in the sense that

$$
E\left(\phi^{n+1}\right) \leq E\left(\phi^{n}\right), \quad \forall n \geq 0 .
$$


Indeed, taking the inner product of (4.7) with $\phi^{n+1}-\phi^{n}$ and using the identity (3.9), we find

$$
\begin{aligned}
\frac{1}{\delta t}\left\|\phi^{n+1}-\phi^{n}\right\|_{0}^{2}+ & \frac{1}{2}\left(\left\|\nabla \phi^{n+1}\right\|_{0}^{2}-\left\|\nabla \phi^{n}\right\|_{0}^{2}+\left\|\nabla\left(\phi^{n+1}-\phi^{n}\right)\right\|_{0}^{2}\right) \\
& +\frac{1}{\varepsilon^{2}}\left(f\left(\phi^{n}\right), \phi^{n+1}-\phi^{n}\right)=0 .
\end{aligned}
$$

For the last term in (4.11), we use the Taylor expansion

$$
F\left(\phi^{n+1}\right)-F\left(\phi^{n}\right)=f\left(\phi^{n}\right)\left(\phi^{n+1}-\phi^{n}\right)+\frac{f^{\prime}\left(\xi^{n}\right)}{2}\left(\phi^{n+1}-\phi^{n}\right)^{2} .
$$

Therefore, by using (4.8), we find

$$
\begin{aligned}
\frac{1}{\delta t}\left\|\phi^{n+1}-\phi^{n}\right\|_{0}^{2} & +\frac{1}{2}\left(\left\|\nabla \phi^{n+1}\right\|_{0}^{2}-\left\|\nabla \phi^{n}\right\|_{0}^{2}+\left\|\nabla\left(\phi^{n+1}-\phi^{n}\right)\right\|_{0}^{2}\right) \\
& +\frac{1}{\varepsilon^{2}}\left(F\left(\phi^{n+1}\right)-F\left(\phi^{n}\right), 1\right) \\
& =\frac{1}{2 \varepsilon^{2}}\left(f^{\prime}\left(\xi^{n}\right)\left(\phi^{n+1}-\phi^{n}\right), \phi^{n+1}-\phi^{n}\right) \leq \frac{L}{2 \varepsilon^{2}}\left\|\phi^{n+1}-\phi^{n}\right\|_{0}^{2},
\end{aligned}
$$

which implies the energy stability.

The condition (4.10) is quite restrictive when $\varepsilon<<1$. To remove this restriction, an implicit treatment for the nonlinear term is usually necessary. Following [17], a simple, yet unconditionally energy stable, second-order scheme for the Allen-Cahn equation is as follows:

$$
\begin{aligned}
& \frac{\phi^{n+1}-\phi^{n}}{\delta t}-\Delta \frac{\phi^{n+1}+\phi^{n}}{2}+\frac{1}{\varepsilon^{2}} \tilde{f}\left(\phi^{n+1}, \phi^{n}\right)=0, \\
& \left.\frac{\partial}{\partial n} \phi^{n+1}\right|_{\partial \Omega}=0
\end{aligned}
$$

where

$$
\tilde{f}(\phi, \psi)= \begin{cases}\frac{F(\phi)-F(\psi)}{\phi-\psi} & \text { if } \phi \neq \psi \\ f(\phi) & \text { if } \phi=\psi\end{cases}
$$

Similarly, the corresponding scheme for the Cahn-Hilliard equation (4.3) is:

$$
\begin{aligned}
& \frac{\phi^{n+1}-\phi^{n}}{\delta t}=\Delta \frac{w^{n+1}+w^{n}}{2},\left.\quad \frac{\partial}{\partial n} w^{n+1}\right|_{\partial \Omega}=0, \\
& \frac{w^{n+1}+w^{n}}{2}=-\Delta \frac{\phi^{n+1}+\phi^{n}}{2}+\frac{1}{\varepsilon^{2}} \tilde{f}\left(\phi^{n+1}, \phi^{n}\right)=0,\left.\quad \frac{\partial}{\partial n} \phi^{n+1}\right|_{\partial \Omega}=0 .
\end{aligned}
$$

It is clear that the above schemes are second-order accurate since they are based on a modified Crank-Nicolson scheme. To show that they are 
unconditionally energy stable, let us consider the scheme (4.13) and take the inner product with $\phi^{n+1}-\phi^{n}$, we find

$\frac{1}{\delta t}\left\|\phi^{n+1}-\phi^{n}\right\|^{2}+\frac{1}{2}\left(\left\|\nabla \phi^{n+1}\right\|^{2}-\left\|\nabla \phi^{n}\right\|^{2}\right)+\frac{1}{\varepsilon^{2}}\left(F\left(\phi^{n+1}\right)-F\left(\phi^{n}\right), 1\right)=0$,

which is exactly

$$
E\left(\phi^{n+1}\right)=E\left(\phi^{n}\right)-\frac{1}{\delta t}\left\|\phi^{n+1}-\phi^{n}\right\|^{2} .
$$

Similar result can be established for the scheme (4.15).

While the scheme (4.13) enjoys the second-order accuracy and unconditional energy stability, it is however not very convenient in practice since (i) a non-linear equation has to be solved at each time step; (ii) the non-linear equation may have multiple solutions; and (iii) the unique solvability can only be guaranteed for $\delta t$ sufficiently small.

We shall present below two classes of schemes which are more suitable in practice.

\subsection{Convex splitting schemes}

The idea of convex splitting was first introduced by Eyre [21]. Recently, the idea has been applied to various gradient flows (cf. [74, 73]). Assuming that we can split $F(\phi)$ as the difference of two convex functions, i.e.,

$$
F(\phi)=F_{c}(\phi)-F_{e}(\phi) \quad \text { with } \quad F_{c}^{\prime \prime}(\phi), F_{e}^{\prime \prime}(\phi) \geq 0 .
$$

Then, the first-order convex splitting scheme reads:

$$
\begin{aligned}
& \frac{\phi^{n+1}-\phi^{n}}{\delta t}-\Delta \phi^{n+1}+\frac{1}{\varepsilon^{2}}\left(f_{c}\left(\phi^{n+1}\right)-f_{e}\left(\phi^{n}\right)\right)=0, \\
& \left.\frac{\partial}{\partial n} \phi^{n+1}\right|_{\partial \Omega}=0,
\end{aligned}
$$

where $f_{c}(\phi)=F_{c}^{\prime}(\phi)$ and $f_{e}(\phi)=F_{e}^{\prime}(\phi)$.

Theorem 4.1: The scheme (4.18) is unconditionally energy stable. More precisely, we have

$$
E\left(\phi^{n+1}\right) \leq E\left(\phi^{n}\right)-\frac{1}{\delta t}\left\|\phi^{n+1}-\phi^{n}\right\|^{2}-\frac{1}{2}\left\|\nabla\left(\phi^{n+1}-\phi^{n}\right)\right\|^{2} .
$$

Furthermore, the solution $\phi^{n+1}$ of the nonlinear equation (4.18) is the unique minimizer of the convex functional

$$
Q(\phi)=\int_{\Omega}\left(\frac{1}{\delta t}|\phi|^{2}+\frac{1}{2}|\nabla \phi|^{2}+\frac{1}{\varepsilon^{2}} F_{c}(\phi)-g^{n} \phi\right) d x,
$$

where $g^{n}=-\frac{1}{\delta t} \phi^{n}-\frac{1}{\varepsilon^{2}} f_{e}\left(\phi^{n}\right)$. 
Proof: Taking the inner product of (4.18) with $\phi^{n+1}-\phi^{n}$, and using the Taylor expansion

$$
\begin{aligned}
& F_{c}\left(\phi^{n}\right)-F_{c}\left(\phi^{n+1}\right)=F_{c}^{\prime}\left(\phi^{n+1}\right)\left(\phi^{n}-\phi^{n+1}\right)+\frac{F_{c}^{\prime \prime}\left(\xi_{n}\right)}{2}\left(\phi^{n}-\phi^{n+1}\right)^{2}, \\
& F_{c}\left(\phi^{n+1}\right)-F_{c}\left(\phi^{n}\right)=F_{c}^{\prime}\left(\phi^{n}\right)\left(\phi^{n+1}-\phi^{n}\right)+\frac{F_{c}^{\prime \prime}\left(\eta_{n}\right)}{2}\left(\phi^{n+1}-\phi^{n}\right)^{2},
\end{aligned}
$$

thanks to (4.17), we find that

$$
\begin{aligned}
\frac{1}{\delta t}\left\|\phi^{n+1}-\phi^{n}\right\|^{2} & +\frac{1}{2}\left(\left\|\nabla \phi^{n+1}\right\|^{2}-\left\|\nabla \phi^{n}\right\|^{2}+\left\|\nabla\left(\phi^{n+1}-\phi^{n}\right)\right\|^{2}\right) \\
& +\frac{1}{\varepsilon^{2}}\left[\left(F\left(\phi^{n+1}\right), 1\right)-\left(F\left(\phi^{n}\right), 1\right)\right] \\
& =-\frac{1}{2 \varepsilon^{2}}\left(F_{c}^{\prime \prime}\left(\xi_{n}\right)+F_{c}^{\prime \prime}\left(\eta_{n}\right),\left(\phi^{n}-\phi^{n+1}\right)^{2}\right) \leq 0
\end{aligned}
$$

which implies (4.19).

On the other hand, it is clear that (4.18) is the Euler-Lagrange equation of $\min _{\phi \in H^{1}(\Omega)} Q(\phi)$. Since $Q(\phi)$ is convex, $\phi^{n+1}$ is its unique minimizer.

One can also construct second-order convex splitting schemes. However, there does not seem to be a second-order convex splitting scheme for general $F(\phi)=F_{c}(\phi)-F_{e}(\phi)$. Its construction depends on the exact form of $F(\phi)$. For $F(\phi)=\frac{1}{4}\left(\phi^{2}-1\right)^{2}$ and $f(\phi)=F^{\prime}(\phi)=\phi^{3}-\phi$, a second-order convex splitting scheme is as follows (cf. [37]):

$$
\begin{aligned}
\frac{\phi^{n+1}-\phi^{n}}{\delta t}-\Delta \frac{\phi^{n+1}+\phi^{n}}{2}+\frac{1}{4 \varepsilon^{2}}( & \left.\left(\phi^{n+1}\right)^{2}+\left(\phi^{n}\right)^{2}\right)\left(\phi^{n+1}+\phi^{n}\right) \\
& -\frac{1}{2 \varepsilon^{2}}\left(3 \phi^{n}-\phi^{n-1}\right)=0 \\
\left.\frac{\partial}{\partial n} \phi^{n+1}\right|_{\partial \Omega} & =0
\end{aligned}
$$

Theorem 4.2: The scheme (4.21) is unconditionally stable. More precisely, we have

$E\left(\phi^{n+1}\right)+\frac{1}{4 \varepsilon^{2}}\left\|\phi^{n+1}-\phi^{n}\right\|^{2} \leq E\left(\phi^{n}\right)+\frac{1}{4 \varepsilon^{2}}\left\|\phi^{n}-\phi^{n-1}\right\|^{2}-\frac{1}{\delta t}\left\|\phi^{n+1}-\phi^{n}\right\|^{2}$.

Furthermore, (4.21) admits a unique solution which is the minimizer of a convex functional. 
Proof: We take the inner product of (4.21) with $\phi^{n+1}-\phi^{n}$. Since

$$
\begin{aligned}
\left(3 \phi^{n}-\phi^{n-1}, \phi^{n+1}-\phi^{n}\right)=\left(\phi^{n+1}+\phi^{n}-\left(\phi^{n+1}-2 \phi^{n}+\phi^{n-1}\right), \phi^{n+1}-\phi^{n}\right) & \\
& =\left(\left(\phi^{n+1}\right)^{2}-\left(\phi^{n}\right)^{2}, 1\right) \\
& -\frac{1}{2}\left(\left\|\phi^{n+1}-\phi^{n}\right\|^{2}-\left\|\phi^{n}-\phi^{n-1}\right\|^{2}+\left\|\phi^{n+1}-2 \phi^{n}+\phi^{n-1}\right\|^{2}\right),
\end{aligned}
$$

we obtain

$$
\begin{aligned}
& \frac{1}{\delta t}\left\|\phi^{n+1}-\phi^{n}\right\|^{2}+\frac{1}{2}\left(\left\|\nabla \phi^{n+1}\right\|^{2}-\left\|\nabla \phi^{n}\right\|^{2}\right) \\
& \quad+\frac{1}{4 \varepsilon^{2}}\left(\left(\phi^{n+1}\right)^{4}-\left(\phi^{n}\right)^{4}-2\left(\left(\phi^{n+1}\right)^{2}-\left(\phi^{n}\right)^{2}\right), 1\right) \\
& \quad+\frac{1}{4 \varepsilon^{2}}\left(\left\|\phi^{n+1}-\phi^{n}\right\|^{2}-\left\|\phi^{n}-\phi^{n-1}\right\|^{2}+\left\|\phi^{n+1}-2 \phi^{n}+\phi^{n-1}\right\|^{2}\right)=0,
\end{aligned}
$$

which implies (4.22).

Next, we show that the solution of (4.21) is the unique minimizer of a convex functional. To this end, we rewrite $\phi^{n+1}$ as the solution of

$$
\begin{aligned}
& \frac{\phi}{\delta t}-\frac{1}{2} \Delta \phi+r_{n}(\phi)=g^{n}, \\
& \left.\frac{\partial}{\partial n} \phi\right|_{\partial \Omega}=0,
\end{aligned}
$$

where

$$
\begin{aligned}
& r_{n}(\phi)=\frac{1}{4 \varepsilon^{2}}\left((\phi)^{2}+\left(\phi^{n}\right)^{2}\right)\left(\phi+\phi^{n}\right), \\
& g^{n}=\frac{\phi^{n}}{\delta t}+\frac{1}{2} \Delta \phi^{n}+\frac{1}{2 \varepsilon^{2}}\left(3 \phi^{n}-\phi^{n-1}\right) .
\end{aligned}
$$

Setting $R_{n}(\phi)=\int r_{n}(\phi) d \phi$ and

$$
Q(\phi)=\int_{\Omega}\left(\frac{1}{\delta t}+\frac{1}{4}|\nabla \phi|^{2}+R_{n}(\phi)-g^{n} \phi\right) d x,
$$

we find that (4.23) is the Euler-Lagrange equation of $\min _{\phi \in H^{1}(\Omega)} Q(\phi)$. Since

$$
r_{n}^{\prime}(\phi)=\frac{1}{4 \varepsilon^{2}}\left(2 \phi\left(\phi+\phi^{n}\right)+\phi^{2}+\left(\phi^{n}\right)^{2}\right)=\frac{1}{4 \varepsilon^{2}}\left(2 \phi^{2}+\left(\phi+\phi^{n}\right)^{2}\right) \geq 0,
$$

we conclude that $Q(\phi)$ is convex and $\phi^{n+1}$ is its unique minimizer. 


\subsection{Stabilized schemes}

Compared to the implicit schemes such as (4.15), the convex splitting schemes admit a unique solution which is the minimizer of a convex functional. Therefore, they are computationally more efficient than the implicit schemes such as (4.15). However, they still need to solve a nonlinear equation at each time step.

The stabilized first-order semi-implicit method reads

$$
\begin{aligned}
& \left(\frac{1}{\delta t}+\frac{S}{\varepsilon^{2}}\right)\left(\phi^{n+1}-\phi^{n}\right)-\Delta \phi^{n+1}+\frac{1}{\varepsilon^{2}} f\left(\phi^{n}\right)=0, \\
& \left.\frac{\partial}{\partial n} \phi^{n+1}\right|_{\partial \Omega}=0
\end{aligned}
$$

where $S$ is a stabilizing parameter to be specified.

The stabilizing term $\frac{S}{\varepsilon^{2}}\left(\phi^{n+1}-\phi^{n}\right)$ introduces an extra consistency error of order $\frac{S \delta t}{\varepsilon^{2}} u_{t}\left(\xi_{n}\right)$. We note that this error is of the same order as the error introduced by the explicit treatment of the nonlinear term which is, $\frac{1}{\varepsilon^{2}}\left(f\left(u\left(t^{n+1}\right)\right)-f\left(u\left(t^{n}\right)\right)\right)=\frac{\delta t}{\varepsilon^{2}} u_{t}\left(\eta_{n}\right)$. Therefore, the stabilized semiimplicit scheme (4.24) is of the same order of accuracy, in terms of $\delta t$ and $\varepsilon$, as the standard semi-implicit scheme - (4.24) with $S=0$, or the firstorder convex splitting scheme (4.18). However, we have the following result for the stabilized scheme.

Lemma 4.1: Assuming that (4.8) holds. Then, for $S \geq \frac{L}{2}$, the stabilized scheme (4.24) is unconditionally stable and the following energy law holds for any $\delta t$ :

$$
E\left(\phi^{n+1}\right) \leq E\left(\phi^{n}\right), \quad \forall n \geq 0
$$

Proof: Taking the inner product of (4.24) with $\phi^{n+1}-\phi^{n}$, we obtain

$$
\begin{gathered}
\left(\frac{1}{\delta t}+\frac{S}{\varepsilon^{2}}\right)\left\|\phi^{n+1}-\phi^{n}\right\|_{0}^{2}+\frac{1}{2}\left(\left\|\nabla \phi^{n+1}\right\|_{0}^{2}-\left\|\nabla \phi^{n}\right\|_{0}^{2}+\left\|\nabla \phi^{n+1}-\nabla \phi^{n}\right\|_{0}^{2}\right) \\
+\frac{1}{\varepsilon^{2}}\left(f\left(\phi^{n}\right), \phi^{n+1}-\phi^{n}\right)=0 .
\end{gathered}
$$

Using again the Taylor expansion (4.12) and (4.8), we find

$$
\left(f\left(\phi^{n}\right), \phi^{n+1}-\phi^{n}\right) \geq\left(F\left(\phi^{n+1}\right)-F\left(\phi^{n}\right), 1\right)-\frac{L}{2}\left(\phi^{n+1}-\phi^{n}, \phi^{n+1}-\phi^{n}\right) .
$$

We can then conclude the desired result from the above two relations. 
We now consider the first-order stabilized semi-implicit method for the Cahn-Hilliard equation (4.3):

$$
\begin{gathered}
\frac{1}{\delta t}\left(\phi^{n+1}-\phi^{n}\right)-\Delta w^{n+1}=0,\left.\frac{\partial w^{n+1}}{\partial n}\right|_{\partial \Omega}=0 \\
-\Delta \phi^{n+1}+\frac{S}{\varepsilon^{2}}\left(\phi^{n+1}-\phi^{n}\right)+\frac{1}{\varepsilon^{2}} f\left(\phi^{n}\right)=w^{n+1},\left.\frac{\partial \phi^{n+1}}{\partial n}\right|_{\partial \Omega}=0 .
\end{gathered}
$$

As in the Allen-Cahn case, the extra consistency error introduced by the stabilization term is of the same order, in terms of $\delta t$ and $\varepsilon$, as the dominating truncation error in the standard semi-implicit scheme, (4.26) with $S=0$.

Lemma 4.2: For $S \geq \frac{L}{2}$, the stabilized scheme (4.26) is unconditionally stable and the following energy law holds for any $\delta t$ :

$$
E\left(\phi^{n+1}\right) \leq E\left(\phi^{n}\right) \quad \forall n \geq 0 .
$$

Proof: Taking the inner product of (4.26a) with $\delta t w^{n+1}$ and that of (4.26b) with $\phi^{n+1}-\phi^{n}$, and using (4.12), we find

$$
\left(\phi^{n+1}-\phi^{n}, w^{n+1}\right)+\delta t\left\|\nabla w^{n+1}\right\|_{0}^{2}=0,
$$

and

$$
\begin{aligned}
\frac{1}{2}\left(\left\|\nabla \phi^{n+1}\right\|_{0}^{2}\right. & \left.-\left\|\nabla \phi^{n}\right\|_{0}^{2}+\left\|\nabla\left(\phi^{n+1}-\phi^{n}\right)\right\|_{0}^{2}\right) \\
& +\frac{S}{\varepsilon^{2}}\left\|\phi^{n+1}-\phi^{n}\right\|_{0}^{2}+\frac{1}{\varepsilon^{2}}\left(F\left(\phi^{n+1}\right)-F\left(\phi^{n}\right), 1\right) \\
& -\frac{1}{2 \varepsilon^{2}}\left(f^{\prime}\left(\xi^{n}\right)\left(\phi^{n+1}-\phi^{n}\right), \phi^{n+1}-\phi^{n}\right)=\left(w^{n+1}, \phi^{n+1}-\phi^{n}\right) .
\end{aligned}
$$

On the other hand, taking the inner product of $(4.26 \mathrm{a})$ with $\sqrt{2 \delta t}\left(\phi^{n+1}-\right.$ $\left.\phi^{n}\right)$, we obtain

$$
\begin{aligned}
\sqrt{\frac{2}{\delta t}}\left\|\phi^{n+1}-\phi^{n}\right\|_{0}^{2} & =-\sqrt{2 \delta t}\left(\nabla w^{n+1}, \nabla\left(\phi^{n+1}-\phi^{n}\right)\right) \\
& \leq \delta t\left\|\nabla w^{n+1}\right\|_{0}^{2}+\frac{1}{2}\left\|\nabla\left(\phi^{n+1}-\phi^{n}\right)\right\|_{0}^{2} .
\end{aligned}
$$

Summing up the above three relations and using (4.8), we arrive at

$$
\begin{aligned}
\sqrt{\frac{2}{\delta t}\left\|\phi^{n+1}-\phi^{n}\right\|_{0}^{2}} & +\frac{S}{\varepsilon^{2}}\left\|\phi^{n+1}-\phi^{n}\right\|_{0}^{2}+\frac{\delta t}{2}\left\|\nabla w^{n+1}\right\|_{0}^{2} \\
& +\frac{1}{2}\left(\left\|\nabla \phi^{n+1}\right\|_{0}^{2}-\left\|\nabla \phi^{n}\right\|_{0}^{2}\right)+\frac{1}{\varepsilon^{2}}\left(F\left(\phi^{n+1}\right)-F\left(\phi^{n}\right), 1\right) \\
& =\frac{1}{2 \varepsilon^{2}}\left(f^{\prime}\left(\xi^{n}\right)\left(\phi^{n+1}-\phi^{n}\right), \phi^{n+1}-\phi^{n}\right) \\
& \leq \frac{L}{2 \varepsilon^{2}}\left\|\phi^{n+1}-\phi^{n}\right\|_{0}^{2} .
\end{aligned}
$$


We conclude that the desired result holds under the condition $S \geq \frac{L}{2}$.

A second-order semi-implicit scheme based on the second-order BNF and Adam-Bash forth is as follows:

$$
\begin{aligned}
& \frac{1}{2 \delta t}\left(3 \phi^{n+1}-4 \phi^{n}+\phi^{n-1}\right)-\Delta \phi^{n+1}+\frac{1}{\varepsilon^{2}}\left(2 f\left(\phi^{n}\right)-f\left(\phi^{n-1}\right)\right)=0, \\
& \left.\frac{\partial \phi^{n+1}}{\partial n}\right|_{\partial \Omega}=0 .
\end{aligned}
$$

Its stabilized version reads:

$$
\begin{aligned}
& \frac{1}{2 \delta t}\left(3 \phi^{n+1}-4 \phi^{n}+\phi^{n-1}\right)+\frac{S}{\varepsilon^{2}}\left(\phi^{n+1}-2 \phi^{n}+\phi^{n-1}\right)-\Delta \phi^{n+1} \\
& \quad+\frac{1}{\varepsilon^{2}}\left(2 f\left(\phi^{n}\right)-f\left(\phi^{n-1}\right)\right)=0,\left.\quad \frac{\partial \phi^{n+1}}{\partial n}\right|_{\partial \Omega}=0 .
\end{aligned}
$$

The stabilizing term $\frac{S}{\varepsilon^{2}}\left(\phi^{n+1}-2 \phi^{n}+\phi^{n-1}\right)$ introduces an extra consistency error of order $\frac{S \delta t^{2}}{\varepsilon^{2}} u_{t t}\left(\xi_{n}\right)$ which is of the same order as the error introduced by replacing $f\left(\phi^{n+1}\right)$ with $2 f\left(\phi^{n}\right)-f\left(\phi^{n-1}\right)$. Therefore, the stabilized semi-implicit scheme (4.29) is of the same order of accuracy, in terms of $\delta t$ and $\varepsilon$, as the semi-implicit scheme (4.28).

The following stability results for the scheme (4.28) and its stabilized version (4.29) are proved in [65].

Lemma 4.3: Under the condition

$$
\delta t \leq \frac{2 \varepsilon^{2}}{3 L}
$$

the solution of the scheme (4.29) with $S \geq 0$ satisfies

$$
\begin{aligned}
E\left(\phi^{n+1}\right) & +\left(\frac{1}{4 \delta t}+\frac{S+L}{2 \varepsilon^{2}}\right)\left\|\phi^{n+1}-\phi^{n}\right\|_{0}^{2} \\
& \leq E\left(\phi^{n}\right)+\left(\frac{1}{4 \delta t}+\frac{S+L}{2 \varepsilon^{2}}\right)\left\|\phi^{n}-\phi^{n-1}\right\|_{0}^{2}, \quad \forall n \geq 1 .
\end{aligned}
$$

Remark 4.1: The above lemma is valid for all $S \geq 0$ which include in particular the usual second-order semi-implicit scheme $(S=0)$. The stability condition (4.30) is only slightly more restrictive than the condition (4.10) for the first-order semi-implicit scheme (4.7).

Unlike in the first-order case, we were unable to show theoretically that the stabilized scheme (4.29) with $S>0$ has better stability than (4.28), which is (4.29) with $S=0$. However, ample numerical evidences indicate that the stabilized version (4.29) with a suitable $S$ allows much large time steps than that is allowed by the unstabilized version (4.28) (cf. [46]). 
Remark 4.2: Beside the two classes of schemes presented above, another class of schemes, namely, the operator splitting schemes [76], are often used to solve Allen-Cahn type equations. While a properly designed operator splitting scheme can also be unconditionally stable and very efficient for the nonlinear reaction-diffusion equations including the Allen-Cahn type equations $[15,14]$, it is not particularly suitable for interface problems that we are interested here, since the diffusion step in an operator splitting scheme does not have any mechanism to keep an interface profile. Furthermore, the operator splitting approach can not be efficiently applied to Cahn-Hilliard type equations.

\section{Numerical schemes for the case of matched density}

In this section, we shall construct simple, yet energy stable numerical schemes for the coupled nonlinear phase-field systems in the case of matched density. To simply the presentation, we shall also assume in this section that the two fluids have the same viscosity $\mu_{0}$ and defer the treatment of variable viscosity to the next section, although the schemes and results in this section extend directly to the case of variable viscosity.

Hereafter, we shall assume that $F(\phi)$ takes the following modified form

$$
F(\phi)= \begin{cases}\frac{1}{\eta^{2}}(\phi-1)^{2}, & \phi>1 \\ \frac{1}{4 \eta^{2}}\left(\phi^{2}-1\right)^{2}, & \phi \in[-1,1], \\ \frac{1}{\eta^{2}}(\phi+1)^{2}, & \phi<-1\end{cases}
$$

which satisfies

$$
\max _{x \in \mathbb{R}}\left|f^{\prime}(x)\right| \leq \frac{2}{\eta^{2}}
$$

\subsection{Allen-Cahn phase-field model}

We consider first the Allen-Cahn phase-field model consisting of the equations (2.10), (2.14), (2.16) with the boundary condition (2.17) and initial condition (2.18).

To fix the idea and simplify the presentation, we shall consider the AllenCahn phase equation (2.10), instead of (2.11) with the Lagrange multiplier $\xi(t)$, as the Lagrange multiplier does not introduce any computational or analytical difficulties.

The term $\Delta \phi \nabla \phi$ in (2.14) is not very convenient in practice, particularly if a finite element method is used. It also introduces additional difficulty for 
proving the discrete energy law, since the test function, $\Delta \phi-f(\phi)$ we used in the continuous case, is usually not in the discrete subspace. Therefore, we shall reformulate (2.14) before constructing numerical schemes. Thanks to $(2.11)$, we can replace $\Delta \phi$ in $(2.14)$ by $\frac{1}{\gamma}\left(\phi_{t}+u \cdot \nabla \phi\right)$ after absorbing the term $(f(\phi)-\xi(t)) \nabla \phi=\nabla(F(\phi)-\xi(t) \phi)$ into $\nabla p$ (but still use the same notation for the modified $p$ ). Therfore, the reformulated Allen-Cahn phase-field model reads:

$$
\begin{aligned}
& \phi_{t}+u \cdot \nabla \phi-\gamma(\Delta \phi-f(\phi))=0 \\
& \rho_{0}\left(u_{t}+(u \cdot \nabla) u\right)-\mu_{0} \Delta u+\nabla p+\frac{\lambda}{\gamma}\left(\phi_{t}+u \cdot \nabla \phi\right) \nabla \phi=0, \\
& \nabla \cdot u=0,
\end{aligned}
$$

together with the boundary condition (2.17) and initial condition (2.18).

Taking the inner product of $(5.3 \mathrm{a})$ with $\frac{\lambda}{\gamma} \phi_{t}$ and that of $(5.3 \mathrm{~b})$ with $u$, we find that the above system satisfies the following energy law

$$
\begin{aligned}
\frac{d}{d t} \int_{\Omega}\left(\frac{1}{2} \rho_{0}|u|^{2}+\frac{\lambda}{2}|\nabla \phi|^{2}\right. & +\lambda F(\phi)) d x \\
& =-\int_{\Omega}\left(\mu_{0}|\nabla u|^{2}+\frac{\lambda}{\gamma}\left|\phi_{t}+u \cdot \nabla \phi\right|^{2}\right) d x .
\end{aligned}
$$

A first-order scheme for the system (5.3) based on the pressurecorrection method (3.6)-(3.7) and the stabilized scheme (4.24) is as follows: Given initial conditions $u^{0}$ and $\phi^{0}$, we compute $\left(\phi^{n+1}, \tilde{u}^{n+1}, u^{n+1}, p^{n+1}\right)$ for $n \geq 0$ by

$$
\begin{gathered}
\left\{\begin{array}{l}
\left(\frac{1}{\delta t}+\frac{S}{\eta^{2}}\right)\left(\phi^{n+1}-\phi^{n}\right)+\left(\tilde{u}^{n+1} \cdot \nabla\right) \phi^{n}-\gamma\left(\Delta \phi^{n+1}-f\left(\phi^{n}\right)\right)=0 \\
\left.\partial_{n} \phi^{n+1}\right|_{\partial \Omega}=0
\end{array}\right. \\
\left\{\begin{array}{l}
\rho_{0}\left(\frac{\tilde{u}^{n+1}-u^{n}}{\delta t}+u^{n} \cdot \nabla \tilde{u}^{n+1}\right)-\mu_{0} \Delta \tilde{u}^{n+1}+\nabla p^{n} \\
+\frac{\lambda}{\gamma}\left(\frac{\phi^{n+1}-\phi^{n}}{\delta t}+\tilde{u}^{n+1} \cdot \nabla \phi^{n}\right) \nabla \phi^{n}=0 \\
\left.\tilde{u}^{n+1}\right|_{\partial \Omega}=0 ; \quad \\
\nabla \cdot u^{n+1}=0 \\
\left.n \cdot u^{n+1}\right|_{\partial \Omega}=0
\end{array}\right. \\
\left\{\begin{array}{l}
\rho_{0} \frac{u^{n+1}-\tilde{u}^{n+1}}{\delta t}+\nabla\left(p^{n+1}-p^{n}\right)=0 \\
\end{array}\right.
\end{gathered}
$$


Remark 5.1: At each time step, the above scheme involves a weakly coupled linear system for $\left(\phi^{n+1}, \tilde{u}^{n+1}\right)$. In fact, if we replace $\tilde{u}^{n+1}$ by $\tilde{u}^{n}$ in (5.5a), then $\phi^{n+1}$ and $\tilde{u}^{n+1}$ are decoupled and can be obtained separately by solving two elliptic equations.

We show below that the above scheme admits a discrete energy law.

Theorem 5.1: For $S \geq \gamma$, the scheme (5.5) is unconditionally stable and satisfies the following discrete energy law:

$$
\begin{aligned}
& {\left[\rho_{0}\left\|u^{n+1}\right\|^{2}+\lambda\left\|\nabla \phi^{n+1}\right\|^{2}+2 \lambda\left(F\left(\phi^{n+1}\right), 1\right)\right]+\frac{\delta t^{2}}{\rho_{0}}\left\|\nabla p^{n+1}\right\|^{2}} \\
& +2 \mu_{0} \delta t\left\|\nabla \tilde{u}^{n+1}\right\|^{2}+\frac{2 \lambda \delta t}{\gamma}\left\|\psi^{n+1}\right\|^{2} \\
& \leq\left[\rho_{0}\left\|u^{n}\right\|^{2}+\lambda\left\|\nabla \phi^{n}\right\|^{2}+2 \lambda\left(F\left(\phi^{n}\right), 1\right)\right]+\frac{\delta t^{2}}{\rho_{0}}\left\|\nabla p^{n}\right\|^{2},
\end{aligned}
$$

where

$$
\psi^{n+1}=\frac{\phi^{n+1}-\phi^{n}}{\delta t}+\tilde{u}^{n+1} \cdot \nabla \phi^{n} .
$$

Proof: Taking the inner product of $(5.5 \mathrm{~b})$ with $2 \delta t \tilde{u}^{n+1}$, we derive

$$
\begin{aligned}
\rho_{0}\left(\left\|\tilde{u}^{n+1}\right\|^{2}\right. & \left.-\left\|u^{n}\right\|^{2}+\left\|\tilde{u}^{n+1}-u^{n}\right\|^{2}\right)+2 \mu_{0} \delta t\left\|\nabla \tilde{u}^{n+1}\right\|^{2} \\
& +2 \delta t\left(\nabla p^{n}, \tilde{u}^{n+1}\right)+\frac{2 \lambda \delta t}{\gamma}\left(\psi^{n+1} \nabla \phi^{n}, \tilde{u}^{n+1}\right)=0 .
\end{aligned}
$$

Taking the inner product of (5.5c) with $\frac{2 \delta t}{\rho_{0}} \nabla p^{n}$, we obtain

$$
\frac{\delta t^{2}}{\rho_{0}}\left(\left\|\nabla p^{n+1}\right\|^{2}-\left\|\nabla p^{n}\right\|^{2}-\left\|\nabla p^{n+1}-\nabla p^{n}\right\|^{2}\right)=2 \delta t\left(\tilde{u}^{n+1}, \nabla p^{n}\right) .
$$

On the other hand, we derive from $(5.5 \mathrm{c})$ that

$$
\frac{\delta t^{2}}{\rho_{0}}\left\|\nabla p^{n+1}-\nabla p^{n}\right\|^{2}=\rho_{0}\left\|\tilde{u}^{n+1}-u^{n+1}\right\|^{2},
$$

and

$$
\left\|u^{n+1}\right\|^{2}+\left\|u^{n+1}-\tilde{u}^{n+1}\right\|^{2}=\left\|\tilde{u}^{n+1}\right\|^{2} .
$$

Combining the above inequalities, we find

$$
\begin{aligned}
\rho_{0}\left(\left\|u^{n+1}\right\|^{2}\right. & \left.-\left\|u^{n}\right\|^{2}+\left\|\tilde{u}^{n+1}-u^{n}\right\|^{2}\right)+2 \mu_{0} \delta t\left\|\nabla \tilde{u}^{n+1}\right\|^{2} \\
& +\frac{\delta t^{2}}{\rho_{0}}\left(\left\|\nabla p^{n+1}\right\|^{2}-\left\|\nabla p^{n}\right\|^{2}\right)+\frac{2 \lambda \delta t}{\gamma}\left(\psi^{n+1} \nabla \phi^{n}, \tilde{u}^{n+1}\right)=0 .
\end{aligned}
$$


Next, taking the inner product of the second equation in (5.5a) with $\frac{2 \lambda}{\gamma}\left(\phi^{n+1}-\phi^{n}\right)$, we find

$$
\begin{aligned}
\frac{2 \lambda \delta t}{\gamma}\left(\psi^{n+1},\right. & \left.\frac{\phi^{n+1}-\phi^{n}}{\delta t}\right)+\frac{2 S \lambda}{\gamma \eta^{2}}\left\|\phi^{n+1}-\phi^{n}\right\|^{2} \\
& +\lambda\left(\left\|\nabla \phi^{n+1}\right\|^{2}-\left\|\nabla \phi^{n}\right\|^{2}+\left\|\nabla \phi^{n+1}-\nabla \phi^{n}\right\|^{2}\right) \\
& +2 \lambda\left(f\left(\phi^{n}\right), \phi^{n+1}-\phi^{n}\right)=0 .
\end{aligned}
$$

We combine (5.11), (5.12) and (4.12) to obtain

$$
\begin{aligned}
\rho_{0}\left(\left\|u^{n+1}\right\|^{2}-\left\|u^{n}\right\|^{2}\right. & \left.+\left\|\tilde{u}^{n+1}-u^{n}\right\|^{2}\right) \\
& +2 \mu_{0} \delta t\left\|\nabla \tilde{u}^{n+1}\right\|^{2}+\frac{\delta t^{2}}{\rho_{0}}\left(\left\|\nabla p^{n+1}\right\|^{2}-\left\|\nabla p^{n}\right\|^{2}\right) \\
& +\frac{2 \lambda \delta t}{\gamma}\left\|\psi^{n+1}\right\|^{2}+\frac{2 S \lambda}{\gamma \eta^{2}}\left\|\phi^{n+1}-\phi^{n}\right\|^{2} \\
& +\lambda\left(\left\|\nabla \phi^{n+1}\right\|^{2}-\left\|\nabla \phi^{n}\right\|^{2}+\left\|\nabla \phi^{n+1}-\nabla \phi^{n}\right\|^{2}\right) \\
& +2 \lambda\left(F\left(\phi^{n+1}\right)-F\left(\phi^{n}\right), 1\right) \\
& \leq \lambda\left(f^{\prime}\left(\xi^{n}\right)\left(\phi^{n+1}-\phi^{n}\right), \phi^{n+1}-\phi^{n}\right) \leq \frac{2 \lambda}{\eta^{2}}\left\|\phi^{n+1}-\phi^{n}\right\|^{2} .
\end{aligned}
$$

We can then conclude from the above inequality.

Remark 5.2: The above stability result and the argument in the proof indicate that if we rewrite the first two coupled equations in (5.5) as a linear system for the unknown $\left(\frac{\lambda}{\gamma}\left(\phi^{n+1}-\phi^{n}\right), \tilde{u}^{n+1}\right)^{t}$, the matrix for the linear system is then positive definite (but not symmetric). Therefore, the coupled system can be efficiently solved by using an interative procedure such as BICGSTAB (cf. for instance [62]). This remark also applies to all other schemes presented below.

\subsection{Cahn-Hilliard phase-field model}

We now consider the Cahn-Hilliard phase-field model consisting of the equations (2.9), (2.14), (2.16).

As in the Allen-Cahn case, the term $\Delta \phi \nabla \phi$ in (2.14) is not convenient in practice. It is also more difficult to treat the fourth-order equation (2.9). Therefore, we split (2.9) into a sequence of two second-order equations as in (4.3), then replace $\Delta \phi \nabla \phi$ in (2.14) by $(-w+f(\phi)) \nabla \phi=-w \nabla \phi+\nabla F(\phi)$, and absorb the last term into $\nabla p$, we arrive at the following equivalent 
system:

$$
\begin{aligned}
& \phi_{t}+u \cdot \nabla \phi=\gamma \Delta w, \\
& w=-\Delta \phi+f(\phi), \\
& \rho_{0}\left(u_{t}+(u \cdot \nabla) u\right)-\mu_{0} \Delta u+\nabla p-\lambda w \nabla \phi=0, \\
& \nabla \cdot u=0,
\end{aligned}
$$

with the boundary condition (2.20) and initial condition (2.18).

Taking the inner product of $(5.13 \mathrm{a})$ with $\lambda w$, that of $(5.13 \mathrm{~b})$ with $\lambda \phi_{t}$ and that of $(5.13 \mathrm{c})$ with 4 , we find that the above system satisfies the following energy law

$$
\begin{aligned}
\frac{d}{d t} \int_{\Omega}\left(\frac{1}{2} \rho_{0}|u|^{2}+\frac{\lambda}{2}|\nabla \phi|^{2}\right. & +\lambda F(\phi)) d x \\
& =-\int_{\Omega}\left(\mu_{0}|\nabla u|^{2}+\lambda \gamma|\nabla w|^{2}\right) d x
\end{aligned}
$$

The following first-order scheme for the system (5.13), based on the pressure-correction projection method $[32,68]$ with a stabilized treatment for the Cahn-Hilliard phase equation [65], was proposed in [64]: Given initial conditions $u^{0}$ and $\phi^{0}$, compute $\left(\phi^{n+1}, w^{n+1}, \tilde{u}^{n+1}, u^{n+1}, p^{n+1}\right)$ for $n \geq 0$ by

$$
\begin{gathered}
\left\{\begin{array}{l}
\frac{1}{\delta t}\left(\phi^{n+1}-\phi^{n}\right)+\left(\tilde{u}^{n+1} \cdot \nabla\right) \phi^{n}=\gamma \Delta w^{n+1}, \\
w^{n+1}-\frac{S}{\eta^{2}}\left(\phi^{n+1}-\phi^{n}\right)=-\Delta \phi^{n+1}+f\left(\phi^{n}\right), \\
\left.\partial_{n} \phi^{n+1}\right|_{\partial \Omega}=0,\left.\quad \partial_{n} w^{n+1}\right|_{\partial \Omega}=0 ;
\end{array}\right. \\
\left\{\begin{array}{l}
\rho_{0}\left(\frac{\tilde{u}^{n+1}-u^{n}}{\delta t}+u^{n} \cdot \nabla \tilde{u}^{n+1}\right)-\mu_{0} \Delta \tilde{u}^{n+1}+\nabla p^{n}-\lambda w^{n+1} \nabla \phi^{n}={ }^{0} \\
\left.\tilde{u}^{n+1}\right|_{\partial \Omega}=0, \\
\left\{\begin{array}{l}
\rho_{0} \frac{u^{n+1}-\tilde{u}^{n+1}}{\delta t}+\nabla\left(p^{n+1}-p^{n}\right)=0, \\
\nabla \cdot u^{n+1}=0, \\
\left.n \cdot u^{n+1}\right|_{\partial \Omega}=0 .
\end{array}\right.
\end{array}\right.
\end{gathered}
$$

Remark 5.3: At each time step, the above scheme involves a weakly coupled linear system for $\left(\phi^{n+1}, w^{n+1}, \tilde{u}^{n+1}\right)$. In fact, if we replace $\tilde{u}^{n+1}$ by $\tilde{u}^{n}$ in $(5.15 \mathrm{a})$, then $\left(\phi^{n+1}, w^{n+1}\right)$ and $\tilde{u}^{n+1}$ are decoupled and can be obtained separately by solving two elliptic equations/systems. 
The following result was proved in [64]:

Theorem 5.2: For $S \geq 1$, the scheme (5.15) is unconditionally stable and satisfies the following discrete energy law:

$$
\begin{aligned}
& {\left[\rho_{0}\left\|u^{n+1}\right\|^{2}+\lambda\left\|\nabla \phi^{n+1}\right\|^{2}+2 \lambda\left(F\left(\phi^{n+1}\right), 1\right)\right]+\frac{\delta t^{2}}{\rho_{0}}\left\|\nabla p^{n+1}\right\|^{2}} \\
& \quad+2 \mu_{0} \delta t\left\|\nabla \tilde{u}^{n+1}\right\|^{2}+2 \lambda \gamma \delta t\left\|\nabla w^{n+1}\right\|^{2} \\
& \leq\left[\rho_{0}\left\|u^{n}\right\|^{2}+\lambda\left\|\nabla \phi^{n}\right\|^{2}+2 \lambda\left(F\left(\phi^{n}\right), 1\right)\right]+\frac{\delta t^{2}}{\rho_{0}}\left\|\nabla p^{n}\right\|^{2} .
\end{aligned}
$$

Proof: The proof is similar to that of Theorem 5.1. For the readers' convenience, we sketch the proof below.

Taking the inner product of $(5.15 \mathrm{~b})$ with $2 \delta t \tilde{u}^{n+1}$, we derive

$$
\begin{aligned}
\rho_{0}\left(\left\|\tilde{u}^{n+1}\right\|^{2}\right. & \left.-\left\|u^{n}\right\|^{2}+\left\|\tilde{u}^{n+1}-u^{n}\right\|^{2}\right)+2 \mu_{0} \delta t\left\|\nabla \tilde{u}^{n+1}\right\|^{2} \\
& +2 \delta t\left(\nabla p^{n}, \tilde{u}^{n+1}\right)-2 \lambda \delta t\left(w^{n+1} \nabla \phi^{n}, \tilde{u}^{n+1}\right)=0 .
\end{aligned}
$$

As in the proof of Theorem 5.1, we have (5.8), (5.9) and (5.10). Combining (5.16), (5.8), (5.9) and (5.10), we find

$$
\begin{aligned}
& \rho_{0}\left(\left\|u^{n+1}\right\|^{2}-\left\|u^{n}\right\|^{2}+\left\|\tilde{u}^{n+1}-u^{n}\right\|^{2}\right)+2 \mu_{0} \delta t\left\|\nabla \tilde{u}^{n+1}\right\|^{2} \\
& +\frac{\delta t^{2}}{\rho_{0}}\left(\left\|\nabla p^{n+1}\right\|^{2}-\left\|\nabla p^{n}\right\|^{2}\right)-2 \lambda \delta t\left(w^{n+1} \nabla \phi^{n}, \tilde{u}^{n+1}\right)=0 .
\end{aligned}
$$

Next, taking the inner product of the first equation in $(5.15 \mathrm{a})$ with $2 \lambda \delta t w^{n+1}$, we get

$$
\begin{aligned}
2 \lambda\left(\phi^{n+1}-\phi^{n}, w^{n+1}\right) & +2 \lambda \delta t\left(\tilde{u}^{n+1} \nabla \phi^{n}, w^{n+1}\right) \\
& +2 \lambda \gamma \delta t\left\|\nabla w^{n+1}\right\|^{2}=0 .
\end{aligned}
$$

Then, taking the inner product of the second equation in (5.15a) with $2 \lambda\left(\phi^{n+1}-\phi^{n}\right)$, we obtain

$$
\begin{aligned}
& 2 \lambda\left(w^{n+1}, \phi^{n+1}-\phi^{n}\right)-\frac{2 \lambda S}{\eta^{2}}\left\|\phi^{n+1}-\phi^{n}\right\|^{2} \\
& =\lambda\left(\left\|\nabla \phi^{n+1}\right\|^{2}-\left\|\nabla \phi^{n}\right\|^{2}+\left\|\nabla \phi^{n+1}-\nabla \phi^{n}\right\|^{2}\right) \\
& +2 \lambda\left(f\left(\phi^{n}\right), \phi^{n+1}-\phi^{n}\right) .
\end{aligned}
$$


Combining (5.17), (5.18), (5.19) and (4.12), we obtain

$$
\begin{aligned}
\rho_{0}\left(\left\|u^{n+1}\right\|^{2}-\left\|u^{n}\right\|^{2}\right. & \left.+\left\|\tilde{u}^{n+1}-u^{n}\right\|^{2}\right) \\
& +2 \mu_{0} \delta t\left\|\nabla \tilde{u}^{n+1}\right\|^{2}+\frac{\delta t^{2}}{\rho_{0}}\left(\left\|\nabla p^{n+1}\right\|^{2}-\left\|\nabla p^{n}\right\|^{2}\right) \\
& +2 \lambda \gamma \delta t\left\|\nabla w^{n+1}\right\|^{2}-\frac{2 S \lambda}{\eta^{2}}\left\|\phi^{n+1}-\phi^{n}\right\|^{2} \\
& +\lambda\left(\left\|\nabla \phi^{n+1}\right\|^{2}-\left\|\nabla \phi^{n}\right\|^{2}+\left\|\nabla \phi^{n+1}-\nabla \phi^{n}\right\|^{2}\right) \\
& +2 \lambda\left(F\left(\phi^{n+1}\right)-F\left(\phi^{n}\right), 1\right) \\
& \leq \lambda\left(f^{\prime}\left(\xi^{n}\right)\left(\phi^{n+1}-\phi^{n}\right), \phi^{n+1}-\phi^{n}\right) \leq \frac{2 \lambda}{\eta^{2}}\left\|\phi^{n+1}-\phi^{n}\right\|^{2} .
\end{aligned}
$$

We can then conclude from the above inequality.

Remark 5.4: One can formally construct second-order stabilized schemes for both the Allen-Cahn Navier-Stokes and Cahn-Hilliard Navier-Stokes models. However, the second-order stabilized schemes do not seem to be unconditionally stable but numerical experiments $[77,65]$ indicate that they allow significantly larger time steps than the non-stabilized schemes.

Remark 5.5: For both the Allen-Cahn and Cahn-Hilliard phase-field models, one can also construct first-order and second-order schemes based on the convex splitting approach for the phase equation and the pressurecorrection for the Navier-Stokes equations. It can be shown as above that such a scheme is unconditionally stable. We leave the detail to the interested readers.

\section{Numerical schemes for the case of variable density and viscosity}

We consider in this section the phase-field models with variable density and viscosity. As described in Section 2, we shall use the modified momentum equation (2.26) which leads to desired energy laws.

\subsection{Allen-Cahn phase-field model}

The Allen-Cahn phase-field model for the case of variable density and viscosity consists of (2.10), (2.26), (2.16) with (2.17). For the sake of simplicity, we use the Allen-Cahn phase equation (2.10) instead of (2.11) with the Lagrange multiplier $\xi(t)$. 
As in the case of matched density and viscosity, the term $\Delta \phi \nabla \phi$ in (2.26) is not suitable for numerical approximation. So we shall reformulate it in a similar fashion and consider the following equivalent system:

$$
\begin{aligned}
& \phi_{t}+u \cdot \nabla \phi=\gamma(\Delta \phi-f(\phi)), \\
& \sigma(\sigma u)_{t}+(\rho u \cdot \nabla) u+\frac{1}{2} \nabla \cdot(\rho u) u-\nabla \cdot \mu D(u)+\nabla p \\
& +\frac{\lambda}{\gamma}\left(\phi_{t}+u \cdot \nabla \phi\right) \nabla \phi=0 \\
& \nabla \cdot u=0 .
\end{aligned}
$$

Taking the inner product of (6.1a) with $\frac{\lambda}{\gamma} \phi_{t}$ and that of $(6.1 \mathrm{~b})$ with $u$, we find that the above system satisfies the following energy law

$$
\begin{aligned}
\frac{d}{d t} \int_{\Omega}\left(\frac{1}{2}|\sigma u|^{2}+\frac{\lambda}{2}|\nabla \phi|^{2}\right. & +\lambda F(\phi)) d x \\
& =-\int_{\Omega}\left(\frac{\mu}{2}|D(u)|^{2}+\frac{\lambda}{\gamma}\left|\phi_{t}+u \cdot \nabla \phi\right|^{2}\right) d x .
\end{aligned}
$$

This coupled nonlinear system presents formidable challenges for algorithm design, analysis and implementation, particularly so when the density ratio is large. We shall present below numerical algorithms which admit a discrete energy law and are easy to solve in practice.

\subsubsection{A scheme based on the Chorin-Temam projection method}

Unlike in the case of matched density and viscosity, it appears difficult to prove stability for a scheme based on the pressure-correction scheme for the case of variable density. Therefore, we shall first present a simple scheme based on the Chorin-Temam projection method. While this scheme lacks sufficient accuracy and is not recommended in practice, it embodies most of the essential ideas in the more accurate schemes that we construct subsequently.

Since the solution of a discretized phase equation does not necessarily satisfy a maximum principle, we set

$$
\hat{\phi}= \begin{cases}\phi, & |\phi| \leq 1 \\ \operatorname{sign}(\phi), & |\phi|>1,\end{cases}
$$

and shall use

$$
\rho(\phi)=\frac{\rho_{1}-\rho_{2}}{2} \hat{\phi}+\frac{\rho_{1}+\rho_{2}}{2}, \mu(\phi)=\frac{\mu_{1}-\mu_{2}}{2} \hat{\phi}+\frac{\mu_{1}+\mu_{2}}{2} .
$$

to update the density and viscosity so as to keep their positivity. 
The scheme based on the Chorin-Temam projection and stabilization reads as follows:

Given initial conditions $u^{0}$ and $\phi^{0}$, we compute $\left(\phi^{n+1}, \tilde{u}^{n+1}, u^{n+1}, p^{n+1}\right)$ for $n \geq 0$ by

$$
\begin{aligned}
& \left\{\begin{array}{l}
\left(\frac{1}{\delta t}+\frac{S}{\eta^{2}}\right)\left(\phi^{n+1}-\phi^{n}\right)+\left(\tilde{u}^{n+1} \cdot \nabla\right) \phi^{n}-\gamma\left(\Delta \phi^{n+1}-f\left(\phi^{n}\right)\right)=0 \\
\left.\frac{\partial}{\partial n} \phi^{n+1}\right|_{\partial \Omega}=0
\end{array}\right. \\
& \left\{\begin{array}{l}
\sigma^{n+1} \frac{\sigma^{n+1} \tilde{u}^{n+1}-\sigma^{n} u^{n}}{\delta t}+\rho^{n}\left(u^{n} \cdot \nabla\right) \tilde{u}^{n+1}+\frac{1}{2}\left(\nabla \cdot\left(\rho^{n} u^{n}\right)\right) \tilde{u}^{n+1} \\
\left.-\nabla \cdot \mu^{n+1} D\left(\tilde{u}^{n+1}\right)+\frac{\lambda}{\gamma}\left(\frac{1}{\delta t}\left(\phi^{n+1}-\phi^{n}\right)+\left(\tilde{u}^{n+1} \cdot \nabla\right) \phi^{n}\right) \nabla \phi^{n}=6.5 \mathrm{~b}\right) \\
\left.\tilde{u}^{n+1}\right|_{\partial \Omega}=0
\end{array}\right.
\end{aligned}
$$

with

$$
\begin{gathered}
\rho^{n+1}=\frac{\rho_{1}-\rho_{2}}{2} \hat{\phi}^{n+1}+\frac{\rho_{1}+\rho_{2}}{2}, \mu^{n+1}=\frac{\mu_{1}-\mu_{2}}{2} \hat{\phi}^{n+1}+\frac{\mu_{1}+\mu_{2}}{2}, \\
\sigma^{n+1}=\sqrt{\rho^{n+1}} ; \\
\qquad\left\{\begin{array}{l}
\rho^{n+1} \frac{u^{n+1}-\tilde{u}^{n+1}}{\delta t}+\nabla p^{n+1}=0, \\
\nabla \cdot u^{n+1}=0, \\
\left.n \cdot u^{n+1}\right|_{\partial \Omega}=0 .
\end{array}\right.
\end{gathered}
$$

Several remarks are in order:

Remark 6.1: From (6.3) and (6.5c), we have $\rho_{1} \leq \rho^{n+1} \leq \rho_{2}$ and $\mu_{1} \leq$ $\mu^{n+1} \leq \mu_{2}$, so $\sigma^{n+1}$ is well defined.

The equations (6.5a)-(6.5b) form a weakly coupled system, for if we replace $\tilde{u}^{n+1}$ by $\tilde{u}^{n}$ in $(6.5 \mathrm{a})$, then we can obtain $\phi^{n+1}$ and $\tilde{u}^{n+1}$ by solving two decoupled linear elliptic equations. The system is only weakly nonlinear through $\sigma^{n+1}$ in $(6.5 \mathrm{~b})$. Thus, it can be efficiently solved by either decoupling the weakly coupled system with a lagged velocity for the convective term in the phase equation, or using a simple sub-iteration process.

Remark 6.2: Equation (6.5d) is decoupled from the other equations. An equivalent formulation is:

$$
\begin{aligned}
& \left(\frac{1}{\rho^{n+1}} \nabla p^{n+1}, \nabla q\right)=\frac{1}{\delta t}\left(\tilde{u}^{n+1}, \nabla q\right), \quad \forall q \in H^{1}(\Omega), \\
& u^{n+1}=\tilde{u}^{n+1}-\delta t \frac{1}{\rho^{n+1}} \nabla p^{n+1} .
\end{aligned}
$$


Thus, an elliptic equation with variable $\frac{1}{\rho^{n+1}}$ needs to be solved at each time step.

We show below that the above scheme admits a discrete energy law.

Theorem 6.1: For $S \geq \gamma$, the solution of scheme (6.5) satisfies the following discrete energy law:

$$
\begin{aligned}
\left\|\sigma^{n+1} u^{n+1}\right\|^{2} & +\lambda\left\|\nabla \phi^{n+1}\right\|^{2}+2 \lambda\left(F\left(\phi^{n+1}\right), 1\right) \\
& +\delta t\left(\frac{2 \lambda}{\gamma}\left\|\psi^{n+1}\right\|^{2}+\left\|\sqrt{\mu^{n+1}} D\left(\tilde{u}^{n+1}\right)\right\|^{2}\right) \\
& \leq\left\|\sigma^{n} u^{n}\right\|^{2}+\lambda\left\|\nabla \phi^{n}\right\|^{2}+2 \lambda\left(F\left(\phi^{n}\right), 1\right),
\end{aligned}
$$

where $\psi^{n+1}$ is defined in (5.6).

Proof: Taking the inner product of $(6.5 \mathrm{~b})$ with $2 \delta t \tilde{u}^{n+1}$, using $(2.28)$ and the fact that

$$
2 \delta t\left(\mu^{n+1} D\left(\tilde{u}^{n+1}\right), \nabla \tilde{u}^{n+1}\right)=\delta t\left\|\sqrt{\mu^{n+1}} D\left(\tilde{u}^{n+1}\right)\right\|^{2},
$$

we derive

$$
\begin{gathered}
\left\|\sigma^{n+1} \tilde{u}^{n+1}\right\|^{2}-\left\|\sigma^{n} u^{n}\right\|^{2}+\left\|\sigma^{n+1} \tilde{u}^{n+1}-\sigma^{n} u^{n}\right\|^{2}+\delta t\left\|\sqrt{\mu^{n+1}} D\left(\tilde{u}^{n+1}\right)\right\|^{2} \\
+\frac{2 \lambda \delta t}{\gamma}\left(\psi^{n+1} \nabla \phi^{n}, \tilde{u}^{n+1}\right)=0
\end{gathered}
$$

Taking the inner product of $(6.5 \mathrm{~d})$ with $2 \delta t u^{n+1}$, we obtain

$$
\left\|\sigma^{n+1} u^{n+1}\right\|^{2}+\left\|\sigma^{n+1}\left(u^{n+1}-\tilde{u}^{n+1}\right)\right\|^{2}=\left\|\sigma^{n+1} \tilde{u}^{n+1}\right\|^{2} .
$$

Combining the above inequalities and using (5.2), we find

$$
\begin{gathered}
\left\|\sigma^{n+1} u^{n+1}\right\|^{2}-\left\|\sigma^{n} u^{n}\right\|^{2}+\left\|\sigma^{n+1}\left(u^{n+1}-\tilde{u}^{n+1}\right)\right\|^{2}+\left\|\sigma^{n+1} \tilde{u}^{n+1}-\sigma^{n} u^{n}\right\|^{2} \\
+\delta t\left\|\sqrt{\mu^{n+1}} D\left(\tilde{u}^{n+1}\right)\right\|^{2}+\frac{2 \lambda \delta t}{\gamma}\left(\psi^{n+1} \nabla \phi^{n}, \tilde{u}^{n+1}\right)=0 .
\end{gathered}
$$

Taking the inner product of $(6.5 \mathrm{a})$ with $\frac{2 \lambda}{\gamma}\left(\phi^{n+1}-\phi^{n}\right)$, we have

$$
\begin{aligned}
\frac{2 \lambda \delta t}{\gamma}\left\|\psi^{n+1}\right\|^{2} & -\frac{2 \lambda \delta t}{\gamma}\left(\psi^{n+1}, \tilde{u}^{n+1} \cdot \nabla \phi^{n}\right)+\frac{2 S \lambda}{\gamma \eta^{2}}\left\|\phi^{n+1}-\phi^{n}\right\|^{2} \\
& +\lambda\left(\left\|\nabla \phi^{n+1}\right\|^{2}-\left\|\nabla \phi^{n}\right\|^{2}+\left\|\nabla \phi^{n+1}-\nabla \phi^{n}\right\|^{2}\right) \\
& +2 \lambda\left(f\left(\phi^{n}\right), \phi^{n+1}-\phi^{n}\right)=0 .
\end{aligned}
$$


For the last term in (6.11), we combine (6.10), (6.11) and (4.12), to obtain

$$
\begin{aligned}
\left\|\sigma^{n+1} u^{n+1}\right\|^{2} & -\left\|\sigma^{n} u^{n}\right\|^{2}+\delta t\left\|\sqrt{\mu^{n+1}} D\left(\tilde{u}^{n+1}\right)\right\|^{2}+\frac{2 \lambda \delta t}{\gamma}\left\|\psi^{n+1}\right\|^{2} \\
& +\frac{2 S \lambda}{\gamma \eta^{2}}\left\|\phi^{n+1}-\phi^{n}\right\|^{2}+\lambda\left(\left\|\nabla \phi^{n+1}\right\|^{2}-\left\|\nabla \phi^{n}\right\|^{2}+\left\|\nabla \phi^{n+1}-\nabla \phi^{n}\right\|^{2}\right) \\
& +2 \lambda\left(F\left(\phi^{n+1}\right)-F\left(\phi^{n}\right), 1\right) \\
& \leq \lambda\left(f^{\prime}\left(\xi^{n}\right)\left(\phi^{n+1}-\phi^{n}\right), \phi^{n+1}-\phi^{n}\right) \leq \frac{2 \lambda}{\eta^{2}}\left\|\phi^{n+1}-\phi^{n}\right\|^{2} .
\end{aligned}
$$

We can then conclude from the above inequality.

\subsubsection{Schemes based on pressure stabilization}

As discussed in Remark 6.2, any scheme with a projection step would result in an elliptic equation with density as the variable coefficient. Therefore, we shall construct efficient schemes based on the pressure stabilized formulation (3.20) (cf., for instance, $[59,68,55,32])$.

The following first-order scheme, originally proposed in [63], is inspired by the incremental pressure stabilization scheme for the Navier-Stokes equations presented in [29].

Given initial conditions $\phi^{0}, p^{0}=0, u^{0}$ and set $\bar{\rho}=\min \left(\rho_{1}, \rho_{2}\right)$. We compute $\left(\phi^{n+1}, u^{n+1}, p^{n+1}\right)$ for $n \geq 0$ by

$$
\begin{aligned}
& \left\{\begin{array}{c}
\left(\frac{1}{\delta t}+\frac{S}{\eta^{2}}\right)\left(\phi^{n+1}-\phi^{n}\right)+\left(u^{n+1} \cdot \nabla\right) \phi^{n}-\gamma\left(\Delta \phi^{n+1}-f\left(\phi^{n}\right)\right)=0 \\
\left.\frac{\partial}{\partial n} \phi^{n+1}\right|_{\partial \Omega}=0 ;
\end{array}\right. \\
& \left\{\begin{array}{c}
\frac{\frac{1}{2}\left(\rho^{n+1}+\rho^{n}\right) u^{n+1}-\rho^{n} u^{n}}{\delta t}+\rho^{n}\left(u^{n} \cdot \nabla\right) u^{n+1}+\frac{1}{2}\left(\nabla \cdot\left(\rho^{n} u^{n}\right)\right) u^{n+1} \\
-\nabla \cdot \mu^{n+1} D\left(u^{n+1}\right)+\nabla\left(2 p^{n}-p^{n-1}\right)
\end{array}\right. \\
& \quad+\frac{\lambda}{\gamma}\left(\frac{\phi^{n+1}-\phi^{n}}{\delta t}+u^{n+1} \cdot \nabla \phi^{n}\right) \nabla \phi^{n}=0 \\
& \left.u^{n+1}\right|_{\partial \Omega}=0 ; \quad
\end{aligned}
$$

with

$$
\rho^{n+1}=\frac{\rho_{1}-\rho_{2}}{2} \hat{\phi}^{n+1}+\frac{\rho_{1}+\rho_{2}}{2}, \mu^{n+1}=\frac{\mu_{1}-\mu_{2}}{2} \hat{\phi}^{n+1}+\frac{\mu_{1}+\mu_{2}}{2} ;
$$




$$
\left\{\begin{array}{l}
\Delta\left(p^{n+1}-p^{n}\right)=\frac{\bar{\rho}}{\delta t} \nabla \cdot u^{n+1}, \\
\left.\frac{\partial}{\partial n} p^{n+1}\right|_{\partial \Omega}=0 .
\end{array}\right.
$$

The above scheme involves a weakly coupled system for $\left(\phi^{n+1}, u^{n+1}\right)$ and a Poisson equation for the pressure increment. So the scheme is particularly attractive for problems with large density ratios.

Theorem 6.2: For $S \geq \gamma$, the solution of the scheme (6.12) satisfies the following energy law:

$$
\begin{aligned}
\left\|\sigma^{n+1} u^{n+1}\right\|^{2} & \left.+\frac{\delta t^{2}}{\bar{\rho}}\left\|\nabla p^{n+1}\right\|^{2}+\lambda\left\|\nabla \phi^{n+1}\right\|^{2}+2 \lambda\left(F\left(\phi^{n+1}\right), 1\right)\right) \\
& +\delta t\left(\frac{2 \lambda}{\gamma}\left\|\psi^{n+1}\right\|^{2}+\left\|\mu^{n+1} D\left(u^{n+1}\right)\right\|^{2}\right) \\
& \leq\left\|\sigma^{n} u^{n}\right\|^{2}+\frac{\delta t^{2}}{\bar{\rho}}\left\|\nabla p^{n}\right\|^{2}+\lambda\left\|\nabla \phi^{n}\right\|^{2}+2 \lambda\left(F\left(\phi^{n}\right), 1\right),
\end{aligned}
$$

where $\sigma^{k}=\sqrt{\rho^{k}}$ and $\psi^{n+1}$ is defined in (5.6).

Proof: Taking the inner product of $(6.12 \mathrm{~b})$ with $2 \delta t u^{n+1}$, from

$$
\begin{aligned}
\left(\frac{1}{2}\left(\rho^{n+1}+\rho^{n}\right) u^{n+1}-\rho^{n} u^{n}, 2 u^{n+1}\right) & =\left\|\sigma^{n+1} u^{n+1}\right\|^{2}-\left\|\sigma^{n} u^{n}\right\|^{2} \\
& +\left\|\sigma^{n}\left(u^{n+1}-u^{n}\right)\right\|^{2},
\end{aligned}
$$

and using (2.28), we have

$$
\begin{aligned}
\left\|\sigma^{n+1} u^{n+1}\right\|^{2} & -\left\|\sigma^{n} u^{n}\right\|^{2}+\left\|\sigma^{n}\left(u^{n+1}-u^{n}\right)\right\|^{2}+\delta t\left\|\sqrt{\mu^{n+1}} D\left(u^{n+1}\right)\right\|^{2} \\
& +2 \delta t\left(p^{n+1}-2 p^{n}+p^{n-1}, \nabla \cdot u^{n+1}\right) \\
& -2 \delta t\left(p^{n+1}, \nabla \cdot u^{n+1}\right)+\frac{2 \lambda \delta t}{\gamma}\left(\psi^{n+1} \nabla \phi^{n}, u^{n+1}\right)=0 .
\end{aligned}
$$

Taking the inner product of $(6.12 \mathrm{c})$ with $\frac{2 \delta t^{2}}{\bar{\rho}}\left(p^{n+1}-2 p^{n}+p^{n-1}\right)$, we obtain

$$
\begin{aligned}
& -\frac{\delta t^{2}}{\bar{\rho}}\left(\left\|\nabla\left(p^{n+1}-p^{n}\right)\right\|^{2}-\left\|\nabla\left(p^{n}-p^{n-1}\right)\right\|^{2}+\left\|\nabla\left(p^{n+1}-2 p^{n}+p^{n-1}\right)\right\|^{2}\right) \\
& =2 \delta t\left(\nabla \cdot u^{n+1}, p^{n+1}-2 p^{n}+p^{n-1}\right)
\end{aligned}
$$

Taking the inner product of $(6.12 \mathrm{c})$ with $-\frac{2 \delta t^{2}}{\bar{\rho}} p^{n+1}$, we obtain

$$
\begin{aligned}
& \frac{\delta t^{2}}{\bar{\rho}}\left(\left\|\nabla p^{n+1}\right\|^{2}-\left\|\nabla p^{n}\right\|^{2}+\| \nabla\left(p^{n+1}-p^{n} \|^{2}\right)\right. \\
& =-2 \delta t\left(\nabla \cdot u^{n+1}, p^{n+1}\right)
\end{aligned}
$$


Adding the above two equalities together, we get

$$
\begin{aligned}
& 2 \delta t\left(p^{n+1}-2 p^{n}+p^{n-1}, \nabla \cdot u^{n+1}\right)-2 \delta t\left(p^{n+1}, \nabla \cdot u^{n+1}\right) \\
& =\frac{\delta t^{2}}{\bar{\rho}}\left(\left\|\nabla p^{n+1}\right\|^{2}-\left\|\nabla p^{n}\right\|^{2}\right)+\frac{\delta t^{2}}{\bar{\rho}}\left\|\nabla\left(p^{n}-p^{n-1}\right)\right\|^{2} \\
& -\frac{\delta t^{2}}{\bar{\rho}}\left\|\nabla\left(p^{n+1}-2 p^{n}+p^{n-1}\right)\right\|^{2} .
\end{aligned}
$$

Taking the difference of (6.12c) at step $n+1$ and step $n$, we derive

$$
\frac{\delta t^{2}}{\bar{\rho}}\left\|\nabla\left(p^{n+1}-2 p^{n}+p^{n-1}\right)\right\|^{2} \leq \bar{\rho}\left\|u^{n+1}-u^{n}\right\|^{2} \leq\left\|\sigma^{n}\left(u^{n+1}-u^{n}\right)\right\|^{2}
$$

Combining the above inequalities together in (6.14), we derive

$$
\begin{aligned}
& \left\|\sigma^{n+1} u^{n+1}\right\|^{2}-\left\|\sigma^{n} u^{n}\right\|^{2}+\delta t\left\|\sqrt{\mu^{n+1}} D\left(u^{n+1}\right)\right\|^{2} \\
& +\frac{\delta t^{2}}{\bar{\rho}}\left(\left\|\nabla p^{n+1}\right\|^{2}-\left\|\nabla p^{n}\right\|^{2}\right)+\frac{\delta t^{2}}{\bar{\rho}}\left\|\nabla\left(p^{n}-p^{n-1}\right)\right\|^{2} \\
& +\frac{2 \lambda \delta t}{\gamma}\left(\psi^{n+1} \nabla \phi^{n}, u^{n+1}\right) \leq 0
\end{aligned}
$$

Taking the inner product of (6.12a) with $\frac{2 \lambda}{\gamma}\left(\phi^{n+1}-\phi^{n}\right)$ and using the same procedure as in the proof of Theorem 5.1, finally we obtain

$$
\begin{aligned}
\left\|\sigma^{n+1} u^{n+1}\right\|^{2} & -\left\|\sigma^{n} u^{n}\right\|^{2}+\delta t\left\|\sqrt{\mu^{n+1}} D\left(u^{n+1}\right)\right\|^{2} \\
& +\frac{\delta t^{2}}{\bar{\rho}}\left(\left\|\nabla p^{n+1}\right\|^{2}-\left\|\nabla p^{n}\right\|^{2}\right)+\frac{\delta t^{2}}{\bar{\rho}}\left\|\nabla\left(p^{n}-p^{n-1}\right)\right\|^{2} \\
& +\frac{2 \lambda \delta t}{\gamma}\left\|\psi^{n+1}\right\|^{2}+\lambda\left(\left\|\nabla \phi^{n+1}\right\|^{2}-\left\|\nabla \phi^{n}\right\|^{2}+\left\|\nabla \phi^{n+1}-\nabla \phi^{n}\right\|^{2}\right) \\
& +2 \lambda\left(F\left(\phi^{n+1}\right)-F\left(\phi^{n}\right), 1\right) \leq 0 .
\end{aligned}
$$

We can also construct a second-order version of the scheme (6.12). More precisely, denoting, for any sequence $\left\{a^{k}\right\}, a^{*, k+1}=2 a^{k}-a^{k-1}$, a secondorder version of $(6.12)$ reads:

$$
\begin{gathered}
\frac{3 \phi^{n+1}-4 \phi^{n}+\phi^{n-1}}{2 \delta t}+\left(u^{n+1} \cdot \nabla\right) \phi^{*, n+1}+\frac{\gamma}{\eta^{2}}\left(\phi^{n+1}-2 \phi^{n}+\phi^{n-1}\right) \\
-\gamma\left(\Delta \phi^{n+1}-2 f\left(\phi^{n}\right)+f\left(\phi^{n-1}\right)\right)=0, \\
\left.\partial_{n} \phi^{n+1}\right|_{\partial \Omega}=0 ;
\end{gathered}
$$




$$
\begin{aligned}
& \frac{\rho^{n+1}}{2 \delta t}\left(3 u^{n+1}-4 u^{n}+u^{n-1}\right)+\rho^{n+1}\left(\nabla \cdot u^{*, n+1}\right) u^{n+1} \\
& \quad-\nabla \cdot \mu^{n+1} D\left(u^{n+1}\right)+\nabla\left(p^{n}+\frac{4}{3} \psi^{n}-\frac{1}{3} \psi^{n-1}\right) \\
& +\frac{\lambda}{\gamma}\left(\frac{1}{2 \delta t}\left(3 \phi^{n+1}-4 \phi^{n}+\phi^{n-1}\right)+\left(u^{n+1} \cdot \nabla\right) \phi^{*, n+1}\right) \nabla \phi^{n+1}=0, \\
& \left.u^{n+1}\right|_{\partial \Omega}=0 ;
\end{aligned}
$$

with

$$
\begin{gathered}
\rho^{n+1}=\frac{\rho_{1}-\rho_{2}}{2} \hat{\phi}^{n+1}+\frac{\rho_{1}+\rho_{2}}{2}, \mu^{n+1}=\frac{\mu_{1}-\mu_{2}}{2} \hat{\phi}^{n+1}+\frac{\mu_{1}+\mu_{2}}{2} \\
\Delta \psi^{n+1}=\frac{3 \bar{\rho}}{2 \delta t} \nabla \cdot u^{n+1} \\
\left.\partial_{n} \psi^{n+1}\right|_{\partial \Omega}=0 \\
p^{n+1}=p^{n}+\psi^{n+1}-\mu^{n+1} \nabla \cdot u^{n+1}
\end{gathered}
$$

We note that the numerical procedure for the above scheme is exactly the same as the scheme (6.12). However, it appears much more difficult to prove that the above scheme satisfies a discrete energy law.

\subsection{Cahn-Hilliard phase-field model}

As in the case of matched density and viscosity, the term $\Delta \phi \nabla \phi$ in (2.26) is not suitable for numerical approximation. So we shall reformulate it in a similar fashion and consider the following equivalent system:

$$
\begin{gathered}
\phi_{t}+u \cdot \nabla \phi=\gamma \Delta w \\
w=-\Delta \phi+f(\phi), \\
\sigma(\sigma u)_{t}+(\rho u \cdot \nabla) u+\frac{1}{2} \nabla \cdot(\rho u) u-\nabla \cdot \mu D(u) \\
+\nabla p-\lambda w \nabla \phi=0, \\
\nabla \cdot u=0 .
\end{gathered}
$$

Taking the inner product of (6.17a) with $\lambda w$, that of (6.17b) with $\lambda \phi_{t}$ and that of $(6.17 \mathrm{c})$ with 4 , we find that the above system satisfies the 
following energy law

$$
\begin{aligned}
\frac{d}{d t} \int_{\Omega}\left(\frac{1}{2}|\sigma u|^{2}+\frac{\lambda}{2}|\nabla \phi|^{2}\right. & +\lambda F(\phi)) d x \\
& =-\int_{\Omega}\left(\frac{\mu}{2}|D(u)|^{2}+\lambda \gamma|\nabla w|^{2}\right) d x
\end{aligned}
$$

Since schemes based on the Chorin-Temam projection method are not sufficiently accurate and efficient, we shall concentrate on constructing schemes based on the pressure-stabilization formulation (cf., for instance, $[59,68,55,32])$, namely, the divergence free condition is replaced by (3.20). The following first-order scheme, similar to (6.12), was proposed in [64] for (6.17):

Given initial conditions $\phi^{0}, p^{0}=0, u^{0}$. We compute $\left(\phi^{n+1}, u^{n+1}, p^{n+1}\right)$ for $n \geq 0$ by

$$
\begin{gathered}
\left\{\begin{array}{l}
\frac{\phi^{n+1}-\phi^{n}}{\delta t}+\left(u^{n+1} \cdot \nabla\right) \phi^{n}=\gamma \Delta w^{n+1} \\
w^{n+1}-\frac{S}{\eta^{2}}\left(\phi^{n+1}-\phi^{n}\right)=-\Delta \phi^{n+1}+f\left(\phi^{n}\right) \\
\left.\partial_{n} \phi^{n+1}\right|_{\partial \Omega}=0,\left.\quad \partial_{n} w^{n+1}\right|_{\partial \Omega}=0
\end{array}\right. \\
\left\{\begin{array}{l}
\frac{\frac{1}{2}\left(\rho^{n+1}+\rho^{n}\right) u^{n+1}-\rho^{n} u^{n}}{\delta t}+\rho^{n}\left(u^{n} \cdot \nabla\right) u^{n+1}+\frac{1}{2}\left(\nabla \cdot\left(\rho^{n} u^{n}\right)\right) u^{n+1} \\
-\nabla \cdot \mu^{n+1} D\left(u^{n+1}\right)+\nabla\left(2 p^{n}-p^{n-1}\right)-\lambda w^{n+1} \nabla \phi^{n}=0 \\
\left.u^{n+1}\right|_{\partial \Omega}=0 ;
\end{array}\right.
\end{gathered}
$$

with

$$
\begin{gathered}
\rho^{n+1}=\frac{\rho_{1}-\rho_{2}}{2} \hat{\phi}^{n+1}+\frac{\rho_{1}+\rho_{2}}{2}, \mu^{n+1}=\frac{\mu_{1}-\mu_{2}}{2} \hat{\phi}^{n+1}+\frac{\mu_{1}+\mu_{2}}{2} . \\
\qquad \begin{array}{l}
\Delta\left(p^{n+1}-p^{n}\right)=\frac{\rho_{1}}{\delta t} \nabla \cdot u^{n+1} \\
\left.\partial_{n} p^{n+1}\right|_{\partial \Omega}=0 .
\end{array}
\end{gathered}
$$

The above scheme only require solving a Poisson equation for the pressure increment, and a weakly coupled system for $\left(\phi^{n+1}, w^{n+1}, u^{n+1}\right)$. So it is particularly suitable for problems with large density ratios.

Theorem 6.3: For $S \geq 1$, the scheme (6.19) is unconditionally stable and 
satisfies the following discrete energy law:

$$
\begin{aligned}
\left\|\sigma^{n+1} u^{n+1}\right\|^{2} & \left.+\frac{\delta t^{2}}{\rho_{1}}\left\|\nabla p^{n+1}\right\|^{2}+\lambda\left\|\nabla \phi^{n+1}\right\|^{2}+2 \lambda\left(F\left(\phi^{n+1}\right), 1\right)\right) \\
& +\delta t\left(2 \lambda \gamma\left\|\nabla w^{n+1}\right\|^{2}+\left\|\sqrt{\mu^{n+1}} D\left(u^{n+1}\right)\right\|^{2}\right) \\
& \leq\left\|\sigma^{n} u^{n}\right\|^{2}+\frac{\delta t^{2}}{\rho_{1}}\left\|\nabla p^{n}\right\|^{2}+\lambda\left\|\nabla \phi^{n}\right\|^{2}+2 \lambda\left(F\left(\phi^{n}\right), 1\right),
\end{aligned}
$$

where $\sigma^{k}=\sqrt{\rho^{k}}$.

Proof: The proof follows essentially the same procedure as in the proof of Theorem 6.2. We sketch the main steps below. For a more detailed proof, we refer to [64].

Taking the inner product of (6.19b) with $2 \delta t u^{n+1}$, using (6.13) and (6.7), we derive

$$
\begin{aligned}
\left\|\sigma^{n+1} u^{n+1}\right\|^{2} & -\left\|\sigma^{n} u^{n}\right\|^{2}+\left\|\sigma^{n}\left(u^{n+1}-u^{n}\right)\right\|^{2}+\delta t\left\|\sqrt{\mu^{n+1}} D\left(u^{n+1}\right)\right\|^{2} \\
& +2 \delta t\left(p^{n+1}-2 p^{n}+p^{n-1}, \nabla \cdot u^{n+1}\right) \\
& -2 \delta t\left(p^{n+1}, \nabla \cdot u^{n+1}\right)-2 \lambda \delta t\left(w^{n+1} \nabla \phi^{n}, u^{n+1}\right)=0 .
\end{aligned}
$$

Observing that the relations (6.14)-(6.16) for the scheme (6.12) are also valid here, we combine (6.20) and (6.16) to obtain

$$
\begin{aligned}
& \left\|\sigma^{n+1} u^{n+1}\right\|^{2}-\left\|\sigma^{n} u^{n}\right\|^{2}+\delta t\left\|\sqrt{\mu^{n+1}} D\left(u^{n+1}\right)\right\|^{2} \\
& +\frac{\delta t^{2}}{\rho_{1}}\left(\left\|\nabla p^{n+1}\right\|^{2}-\left\|\nabla p^{n}\right\|^{2}\right)+\frac{\delta t^{2}}{\rho_{1}}\left\|\nabla\left(p^{n+1}-p^{n}\right)\right\|^{2} \\
& -2 \lambda \delta t\left(w^{n+1} \nabla \phi^{n}, u^{n+1}\right) \leq 0 .
\end{aligned}
$$

Finally, taking the inner product of the first equation in (6.19a) with $2 \lambda \delta t w^{n+1}$, and the second equation in (6.19a) with $2 \lambda\left(\phi^{n+1}-\phi^{n}\right)$ and using the same procedure as in the proof of Theorem 5.2, we can obtain

$$
\begin{aligned}
& \left\|\sigma^{n+1} u^{n+1}\right\|^{2}-\left\|\sigma^{n} u^{n}\right\|^{2}+\delta t\left\|\sqrt{\mu^{n+1}} D\left(u^{n+1}\right)\right\|^{2} \\
& +\frac{\delta t^{2}}{\rho_{1}}\left(\left\|\nabla p^{n+1}\right\|^{2}-\left\|\nabla p^{n}\right\|^{2}\right)+\frac{\delta t^{2}}{\rho_{1}}\left\|\nabla\left(p^{n+1}-p^{n}\right)\right\|^{2} \\
& +2 \lambda \gamma \delta t\left\|\nabla w^{n+1}\right\|^{2}+\lambda\left(\left\|\nabla \phi^{n+1}\right\|^{2}-\left\|\nabla \phi^{n}\right\|^{2}+\left\|\nabla \phi^{n+1}-\nabla \phi^{n}\right\|^{2}\right) \\
& \quad+2 \lambda\left(F\left(\phi^{n+1}\right)-F\left(\phi^{n}\right), 1\right) \leq 0 .
\end{aligned}
$$

As in the Allen-Cahn case, we can construct a second-order version of the scheme (6.19). Still denoting, for any sequence $\left\{a^{k}\right\}, a^{*, k+1}=2 a^{k}-a^{k-1}$, a second-order version of (6.19) reads: 


$$
\begin{aligned}
& \frac{3 \phi^{n+1}-4 \phi^{n}+\phi^{n-1}}{2 \delta t}+\left(u^{n+1} \cdot \nabla\right) \phi^{*, n+1}=\gamma \Delta w^{n+1}, \\
& \left.w^{n+1}-\frac{S}{\eta^{2}}\left(\phi^{n+1}-2 \phi^{n}+\phi^{n-1}\right)=-\Delta \phi^{n+1}+2 f\left(\phi^{n}\right)-f\left(\phi^{n-1}\right), 6.22 \mathrm{a}\right) \\
& \left.\partial_{n} \phi^{n+1}\right|_{\partial \Omega}=0,\left.\quad \partial_{n} w^{n+1}\right|_{\partial \Omega}=0 ; \\
& \frac{\rho^{n+1}}{2 \delta t}\left(3 u^{n+1}-4 u^{n}+u^{n-1}\right)+\rho^{n+1}\left(\nabla \cdot u^{*, n+1}\right) u^{n+1}-\nabla \cdot \mu^{n+1} D\left(u^{n+1}\right) \\
& \quad+\nabla\left(p^{n}+\frac{4}{3} \psi^{n}-\frac{1}{3} \psi^{n-1}\right)-\lambda w^{n+1} \nabla \phi^{n}=0, \quad(6.22 \mathrm{~b}) \\
& \left.u^{n+1}\right|_{\partial \Omega}=0, \quad
\end{aligned}
$$

with

$$
\begin{gathered}
\rho^{n+1}=\frac{\rho_{1}-\rho_{2}}{2} \hat{\phi}^{n+1}+\frac{\rho_{1}+\rho_{2}}{2}, \mu^{n+1}=\frac{\mu_{1}-\mu_{2}}{2} \hat{\phi}^{n+1}+\frac{\mu_{1}+\mu_{2}}{2} ; \\
\Delta \psi^{n+1}=\frac{3 \rho_{1}}{2 \delta t} \nabla \cdot u^{n+1}, \\
\left.\partial_{n} \psi^{n+1}\right|_{\partial \Omega}=0 \\
p^{n+1}=p^{n}+\psi^{n+1}-\mu^{n+1} \nabla \cdot u^{n+1} .
\end{gathered}
$$

Once again, the numerical procedure for the above scheme is exactly the same as the scheme (6.19).

\section{Phase-field models for complex fluids}

The Allen-Cahn and Cahn-Hilliard phase-field models for Newtonian twophase fluids can be extended to deal with various type of complex fluids.

Consider for instance an immiscible blend of a nematic liquid crystal and a Newtonian fluid. There are three types of free energies: mixing energy of the interface, bulk distortion energy of the nematic, and the anchoring energy of the liquid crystal molecules on the interface. As in the Newtonian case, we introduce a phase-field variable $\phi$ such that the concentration of the two components is $(1+\phi) / 2$ and $(1-\phi) / 2$, respectively.

The mixing energy density is still expressed in the Landau-Ginzburg form:

$$
f_{\text {mix }}(\phi, \nabla \phi)=\frac{\lambda}{2}|\nabla \phi|^{2}+\frac{\lambda}{4 \eta^{2}}\left(\phi^{2}-1\right)^{2}
$$


The orientation of the nematic liquid crystal is described by the director field $n(x)$. The Frank distortion energy expresses the energy penalty for distorting the orientation [13]:

$$
f_{\text {bulk }}=K\left[\frac{1}{2} \nabla n:(\nabla n)^{\mathrm{T}}+\frac{\left(|n|^{2}-1\right)^{2}}{4 \delta^{2}}\right],
$$

where $K$ is the elastic constant. The second term on the right-hand side regularized the original Frank energy to allow defects [47]. The nematic prefers to orient on the interface along an easy axis [13]; any deviation from it is penalized by an anchoring energy. Here we assume that the easy axis is any direction in the tangential plane, and write the anchoring energy as

$$
f_{\text {anch }}=\frac{A}{2}(n \cdot \nabla \phi)^{2},
$$

with the positive constant $A$ representing the anchoring strength. This is the diffuse-interface counterpart of the Rapini-Popoular energy [60]. Unlike in the sharp-interface picture, both $f_{m i x}$ and $f_{\text {anch }}$ are volumetric energy densities. Finally, the total free energy density for the two-phase material is written as:

$$
f(\phi, n, \nabla \phi, \nabla n)=f_{m i x}+\frac{1+\phi}{2} f_{b u l k}+f_{\text {anch }}
$$

where $(1+\phi) / 2$ is the volume fraction of the nematic component, and $\phi=1$ in the purely nematic phase.

Variation of the system's action functional with respect to the phasefield variable $\phi$, the nematic director $n$ and the displacement leads to evolution equations for $\phi, n$ and the momentum equation. Augmented by the dissipative effects, the governing equations of the system are:

$$
\begin{aligned}
\frac{\partial \phi}{\partial t}+v \cdot \nabla \phi & =\gamma_{1} \nabla^{2} \frac{\delta F}{\delta \phi} \\
\frac{\partial n}{\partial t}+v \cdot \nabla n & =\gamma_{2} h, \\
\nabla \cdot v & =0, \\
\rho\left(\frac{\partial v}{\partial t}+v \cdot \nabla v\right) & =-\nabla p+\nabla \cdot\left[\mu\left(\nabla v+\nabla v^{T}\right)+\tau^{e}\right],
\end{aligned}
$$

where $\gamma_{1}$ is the interfacial mobility and $\gamma_{2}$ determines the relaxation time for the director field $n . F=\int f d \Omega$ is the total free energy of the system, whose variations produce

$$
\frac{\delta F}{\delta \phi}=\lambda\left[-\nabla^{2} \phi+\frac{\phi\left(\phi^{2}-1\right)}{\epsilon^{2}}\right]+\frac{1}{2} f_{b u l k}-A \nabla \cdot[(n \cdot \nabla \phi) n],
$$


and the molecular field

$$
h=-\frac{\delta F}{\delta n}=K\left[-\nabla \cdot\left(\frac{1+\phi}{2} \nabla n\right)+\frac{1+\phi}{2} \frac{\left(n^{2}-1\right) n}{\delta^{2}}\right]+A(n \cdot \nabla \phi) \nabla \phi .
$$

The last two terms in equation (7.6) represent coupling between the phase field and the Frank distortion energy and anchoring energy. When the interface is thin, $f_{\text {bulk }}$ is dominated by the mixing energy near the interface and therefore negligible. The last term may have an effect on the interfacial $\phi$ profile for strong anchoring. But it is a lower order effect, negligible if the effects of interfacial tension and surface anchoring are assumed to be additive (cf. equation (7.8) below). Thus for simplicity, the last two terms on the right-hand-side of equation (7.6) can often be neglected.

As in the Newtonian case, the elastic stress tensor $\tau^{e}$ can be derived as part of the variational procedure (cf. [78]), and in this case takes the form

$$
\tau^{e}=-\lambda(\nabla \phi \otimes \nabla \phi)-K \frac{1+\phi}{2}(\nabla n) \cdot(\nabla n)^{\mathrm{T}}-A(n \cdot \nabla \phi) n \otimes \nabla \phi .
$$

One can show that in the case of matched density, a solution to the above governing equations obeys the following energy law [78]:

$$
\frac{d}{d t} \int_{\Omega}\left(\frac{\rho}{2}|u|^{2}+f\right) d \Omega=-\int_{\Omega}\left(\mu \nabla u: \nabla u^{\mathrm{T}}+\gamma_{1}\left|\nabla \frac{\delta F}{\delta \phi}\right|^{2}+\gamma_{2}\left|\frac{\delta F}{\delta n}\right|^{2}\right) d \Omega
$$

where $f$ is the system's potential energy density. In the case of variable density, we can modify the momentum equation (7.5d) as in (2.26) so that the above energy law still holds for the modified system.

Although the system (7.5) appears to be much more complicated than its Newtonian counterpart, it is derived using the same energetic variational procedure and satisfies a similar energy law. Thus, one can also design efficient, unconditionally stable coupled energy stable schemes for (7.5) by following the designing principles in the Newtonian case. Alternatively, one can take a decoupled semi-implicit approach where only the principal linear differential operators are treated implicitly while all nonlinear terms are treated explicitly (cf. [46]). In this case, one only needs to solve a sequence of Poisson type equations at each time step. The price for this simplicity is that the scheme is only stable for sufficiently small time steps.

\section{Concluding remarks}

We discussed in this note the phase-field models for two-phase incompressible flows and their numerical approximations. 
The phase-field approach enjoys many advantages as well as suffers from some shortcomings. The notable advantages include: (i) the interface is represented by the level set $\{\phi=0\}$ so there is no need to track the interface; (ii) no special treatment needed for topological changes; (iii) unlike in a level-set approach, the interfacial thickness is preserved by the dynamics of the phase-field models; (iv) it is derived from a energetic variational procedure which ensures that an energy law is available. On the other hand, the main challenges and shortcomings of the phase-field model are: (i) The interface is represented by a smooth curve with a sharp gradient across the interfacial region of thickness $O(\eta)$. A numerical mesh needs to resolve the interfacial region, making it potentially very expensive for $\eta \ll 1$. This difficulty can be alleviated to some extent by using an adaptive mesh or moving mesh method. (ii) The choice of the numerical parameters, such as the interfacial thickness $\eta$ and the time relaxation parameter $\gamma$, is a delicate matter. While it is theoretically more accurate to choose $\eta$ and $\gamma$ as small as possible, but it comes with added numerical costs as decreasing $\eta$ requires more refined mesh to resolve the thinner interfacial region, and decreasing $\gamma$ leads to longer relaxation time scales. We refer to [22] for a more detailed account on these issues.

While we have only discussed the two-phase fluids, the phase-field approach has been extended to treat multi-phase flows with three or more phases (see for instance [42]).

We presented a unified approach on how to design simple, efficient and energy stable time discretization schemes for the coupled nonlinear AllenCahn and Cahn-Hilliard phase-field systems. We placed special emphasis on designing numerical schemes which require solving only linear systems while satisfying discrete energy laws that mimic the energy laws satisfied by the exact solution. Since all of our time discretization schemes can be cast in a weak formulation, they can be used with any consistent Galerkin type spacial discretizations such as finite element methods or spectralGalerkin/spectral-element methods. In fact, the schemes in Sections 5 and 6 have been implemented with a spectral-Galerkin method and used successfully to simulate the dynamics of an air bubble rising in water [63, 64], a particular challenging situation as the density and viscosity ratios are very large.

It is hoped that this note will be useful for researchers interested in modeling and simulation of multi-phase incompressible flows.

Acknowledgment. This work is supported in part by NSF DMS-0915066. 


\section{References}

1. S. M. Allen and J. W. Cahn. A microscopic theory for antiphase boundary motion and its application to antiphase domain coarsening. Acta Metall. Mater., 27:1085-1095, 1979.

2. D. M. Anderson, G. B. McFadden, and A. A. Wheeler. Diffuse-interface methods in fluid mechanics. 30:139-165, 1998.

3. Ivo Babuška. The finite element method with Lagrangian multipliers. Numer. Math., 20:179-192, 1972/73.

4. Roland Becker, Xiaobing Feng, and Andreas Prohl. Finite element approximations of the Ericksen-Leslie model for nematic liquid crystal flow. SIAM J. Numer. Anal., 46(4):1704-1731, 2008.

5. F. Brezzi. On the existence, uniqueness and approximation of saddle-point problems arising from Lagrangian multipliers. Rev. Française Automat. Informat. Recherche Opérationnelle Sér. Rouge, 8(R-2):129-151, 1974.

6. David L. Brown, Ricardo Cortez, and Michael L. Minion. Accurate projection methods for the incompressible Navier-Stokes equations. J. Comput. Phys., 168(2):464-499, 2001.

7. Luis A. Caffarelli and Nora E. Muler. An $L^{\infty}$ bound for solutions of the Cahn-Hilliard equation. Arch. Rational Mech. Anal., 133(2):129-144, 1995.

8. J. W. Cahn and J. E. Hilliard. Free energy of a nonuniform system, i: Interfacial free energy. J. Chem. Phys., 28:258, 1958.

9. Y. C. Chang, T. Y. Hou, B. Merriman, and S. Osher. A level set formulation of eulerian interface capturing methods for incompressible fluid flows. $J$. Comput. Phys., 124(2):449-464, 1996.

10. L.Q. Chen. Phase-field models for microstructure evolution. Annual Review of Material Research, 32:113, 2002.

11. L.Q. Chen and Jie Shen. Applications of semi-implicit Fourier-spectral method to phase-field equations. Comput. Phys. Comm., 108:147-158, 1998.

12. A. J. Chorin. Numerical solution of the Navier-Stokes equations. Math. Comp., 22:745-762, 1968.

13. P. G. de Gennes and J. Prost. The Physics of Liquid Crystals. Oxford University Press, 1993.

14. Stéphane Descombes and Magali Ribot. Convergence of the PeacemanRachford approximation for reaction-diffusion systems. Numer. Math., 95(3):503-525, 2003.

15. Stéphane Descombes and Michelle Schatzman. Strang's formula for holomorphic semi-groups. J. Math. Pures Appl. (9), 81(1):93-114, 2002.

16. Q. Du, C. Liu, and X. Wang. Simulating the deformation of vesicle membranes under elastic bending energy in three dimensions. J. Comput. Phys., 212:757-777, 2005.

17. Qiang Du and R. A. Nicolaides. Numerical analysis of a continuum model of phase transition. SIAM J. Numer. Anal., 28(5):1310-1322, 1991.

18. Weinan E and Jian-Guo Liu. Projection method. I. Convergence and numerical boundary layers. SIAM J. Numer. Anal., 32(4):1017-1057, 1995.

19. Weinan E and Jian-Guo Liu. Projection method. II. Godunov-Ryabenki anal- 
ysis. SIAM J. Numer. Anal., 33(4):1597-1621, 1996.

20. Weinan E and Jian-Guo Liu. Gauge method for viscous incompressible flows. Commun. Math. Sci., 1(2):317-332, 2003.

21. David J. Eyre. Unconditionally gradient stable time marching the CahnHilliard equation. In Computational and mathematical models of microstructural evolution (San Francisco, CA, 1998), volume 529 of Mater. Res. Soc. Sympos. Proc., pages 39-46. MRS, Warrendale, PA, 1998.

22. J. J. Feng, C. Liu, J. Shen, and P. Yue. An energetic variational formulation with phase field methods for interfacial dynamics of complex fluids: advantages and challenges. IMA volumes in Mathematics and Applications, 140:1-26, 2005.

23. Xiaobing Feng, Yinnian He, and Chun Liu. Analysis of finite element approximations of a phase field model for two-phase fluids. Math. Comp., 76(258):539-571 (electronic), 2007.

24. J. Glimm, J. W. Grove, X. L. Li, and D. C. Tan. Robust computational algorithms for dynamic interface tracking in three dimensions. SIAM J. Sci. Comput., 21(6):2240-2256 (electronic), 2000.

25. J. Glimm, X. L. Li, Y. Liu, and N. Zhao. Conservative front tracking and level set algorithms. Proc. Natl. Acad. Sci. USA, 98(25):14198-14201 (electronic), 2001.

26. K. Goda. A multistep technique with implicit difference schemes for calculating two- or three-dimensional cavity flows. J. Comput. Phys., 30:76-95, 1979.

27. J.-L. Guermond. Un résultat de convergence à l'ordre deux en temps pour l'approximation des équations de Navier-Stokes par une technique de projection. Modél. Math. Anal. Numér. (M2AN), 33(1):169-189, 1999.

28. J.-L. Guermond and L. Quartapelle. A projection FEM for variable density incompressible flows. J. Comput. Phys., 165(1):167-188, 2000.

29. J.-L. Guermond and Abner Salgado. A splitting method for incompressible flows with variable density based on a pressure Poisson equation. J. Comput. Phys., 228(8):2834-2846, 2009.

30. J. L. Guermond and Jie Shen. A new class of truly consistent splitting schemes for incompressible flows. J. Comput. Phys., 192(1):262-276, 2003.

31. J. L. Guermond and Jie Shen. Velocity-correction projection methods for incompressible flows. SIAM J. Numer. Anal., 41(1):112-134 (electronic), 2003.

32. J.L. Guermond, P. Minev, and Jie Shen. An overview of projection methods for incompressible flows. Comput. Methods Appl. Mech. Engrg., 195:60116045, 2006.

33. J.L. Guermond and Jie Shen. On the error estimates of rotational pressurecorrection projection methods. Math. Comp, 73:1719-1737, 2004.

34. M. E. Gurtin. Multiphase thermodynamics with interfacial structure, 1. Heat conduction and the capillary balance law. Archive for Rational Mechanics and Analysis, 104:195-221, 1988.

35. M. E. Gurtin, D. Polignone, and J. Viñals. Two-phase binary fluids and immiscible fluids described by an order parameter. Math. Models Methods Appl. Sci., 6(6):815-831, 1996. 
36. Thomas Y. Hou and Brian T. R. Wetton. Second-order convergence of a projection scheme for the incompressible Navier-Stokes equations with boundaries. SIAM J. Numer. Anal., 30(3):609-629, 1993.

37. Z. Hu, S. M. Wise, C. Wang, and J. S. Lowengrub. Stable and efficient finitedifference nonlinear-multigrid schemes for the phase field crystal equation. $J$. Comput. Phys., 228(15):5323-5339, 2009.

38. D. Jacqmin. Diffuse interface model for incompressible two-phase flows with large density ratios. J. Comput. Phys., 155(1):96-127, 2007.

39. Hans Johnston and Jian-Guo Liu. Accurate, stable and efficient NavierStokes solvers based on explicit treatment of the pressure term. J. Comput. Phys., 199(1):221-259, 2004.

40. G. E. Karniadakis, M. Israeli, and S. A. Orszag. High-order splitting methods for the incompressible Navier-Stokes equations. J. Comput. Phys., 97:414443, 1991.

41. J. Kim and P. Moin. Application of a fractional-step method to incompressible Navier-Stokes equations. J. Comput. Phys., 59:308-323, 1985.

42. Junseok Kim and John Lowengrub. Phase field modeling and simulation of three-phase flows. Interfaces Free Bound., 7(4):435-466, 2005.

43. J. Li and Y. Renardy. Numerical study of flows of two immiscible liquids at low reynolds number. SIAM Review, 42:417-439, 2000.

44. J. Li and Y. Renardy. Shear-induced rupturing of a viscous drop in a bingham liquid. J. Non-Newtonian Fluid Mech., 95:235-251, 2000.

45. Ping Lin, Chun Liu, and Hui Zhang. An energy law preserving $C^{0}$ finite element scheme for simulating the kinematic effects in liquid crystal dynamics. J. Comput. Phys., 227(2):1411-1427, 2007.

46. C. Liu, J. Shen, and X. Yang. Dynamics of defect motion in nematic liquid crystal flow: Modeling and numerical simulation. Commun. Comput. Phys., 2:1184-1198, 2007.

47. C. Liu and N. J. Walkington. Approximation of liquid crystal flows. SIAM J. Numer. Anal, 37:725-741, 2000.

48. Chun Liu and Jie Shen. A phase field model for the mixture of two incompressible fluids and its approximation by a Fourier-spectral method. Physica D, 179(3-4):211-228, 2003.

49. Jian-Guo Liu, Jie Liu, and Robert L. Pego. Stability and convergence of efficient Navier-Stokes solvers via a commutator estimate. Comm. Pure Appl. Math., 60(10):1443-1487, 2007.

50. J. Lowengrub and L. Truskinovsky. Quasi-incompressible Cahn-Hilliard fluids and topological transitions. R. Soc. Lond. Proc. Ser. A Math. Phys. Eng. Sci., 454(1978):2617-2654, 1998.

51. Ricardo H. Nochetto and Jae-Hong Pyo. Error estimates for semi-discrete gauge methods for the Navier-Stokes equations. Math. Comp., 74(250):521542 (electronic), 2005.

52. S. A. Orszag, M. Israeli, and M. Deville. Boundary conditions for incompressible flows. J. Sci. Comput., 1:75-111, 1986.

53. S. Osher and J. Sethian. Fronts propagating with curvature dependent speed: Algorithms based on Hamilton Jacobi formulations. Journal of Computa- 
tional Physics, 79:12-49, 1988.

54. J. Blair Perot. An analysis of the fractional step method. J. Comput. Phys., 108(1):51-58, 1993.

55. Andreas Prohl. Projection and quasi-compressibility methods for solving the incompressible Navier-Stokes equations. Advances in Numerical Mathematics. B. G. Teubner, Stuttgart, 1997.

56. Jae-Hong Pyo and Jie Shen. Gauge-Uzawa methods for incompressible flows with variable density. J. Comput. Phys., 221(1):181-197, 2007.

57. Tiezheng Qian, Xiao-Ping Wang, and Ping Sheng. A variational approach to moving contact line hydrodynamics. J. Fluid Mech., 564:333-360, 2006.

58. Alfio Quarteroni, Fausto Saleri, and Alessandro Veneziani. Factorization methods for the numerical approximation of Navier-Stokes equations. Comput. Methods Appl. Mech. Engrg., 188(1-3):505-526, 2000.

59. R. Rannacher. On Chorin's projection method for the incompressible NavierStokes equations. Lecture Notes in Mathematics, vol. 1530, 1991.

60. A. Rapini and M. Popoular. Distortion d'une lamelle nematique sous champ magnetique conditions d'ancrage aux parois. J. Phys. (Paris) C, 30:54-56, 1969.

61. Lord Rayleigh. On the theory of surface forces ii. Phil. Mag., 33:209, 1892.

62. Yousef Saad. Iterative methods for sparse linear systems. Society for Industrial and Applied Mathematics, Philadelphia, PA, second edition, 2003.

63. J. Shen and X. Yang. A phase-field model and its numerical approximation for two-phase incompressible flows with different densities and viscosities. SIAM J. Sci. Comput., 32:1159-1179, 2010.

64. J. Shen and X. Yang. Energy stable schemes for cahn-hilliard phase-field model of two phase incompressible flows equations. Chinese Annals of Mathematics, Series B, 2010, DOI: 10.1007/s11401-010-0599-y.

65. J. Shen and X. Yang. Numerical approximations of allen-cahn and cahnhilliard equations. Discrete and Continuous Dynamical Systems. Series A, 2010, to appear.

66. Jie Shen. On error estimates of the projection methods for the Navier-Stokes equations: first-order schemes. SIAM J. Numer. Anal., 29:57-77, 1992.

67. Jie Shen. A new pseudo-compressibility method for the Navier-Stokes equations. Appl. Numer. Math., 21:71-90, 1996.

68. Jie Shen. On error estimates of projection methods for the Navier-Stokes equations: second-order schemes. Math. Comp, 65:1039-1065, July 1996.

69. R. Temam. Une méthode d'approximation des solutions des équations de Navier-Stokes. Bull. Soc. Math. France, 98:115-152, 1968.

70. L. J. P. Timmermans, P. D. Minev, and F. N. Van De Vosse. An approximate projection scheme for incompressible flow using spectral elements. Int. J. Numer. Methods Fluids, 22:673-688, 1996.

71. J. van der Waals. The thermodynamic theory of capillarity under the hypothesis of a continuous density variation. J. Stat. Phys., 20:197-244, 1893.

72. J. van Kan. A second-order accurate pressure-correction scheme for viscous incompressible flow. SIAM J. Sci. Stat. Comput., 7:870-891, 1986.

73. Cheng Wang, Xiaoming Wang, and Steven M. Wise. Unconditionally sta- 
ble schemes for equations of thin film epitaxy. Discrete Contin. Dyn. Syst., 28(1):405-423, 2010.

74. S. M. Wise, C. Wang, and J. S. Lowengrub. An energy-stable and convergent finite-difference scheme for the phase field crystal equation. SIAM J. Numer. Anal., 47(3):2269-2288, 2009.

75. Chuanju Xu and Tao Tang. Stability analysis of large time-stepping methods for epitaxial growth models. SIAM J. Numer. Anal., 44(4):1759-1779, 2006.

76. N. N. Yanenko. Splitting methods for partial differential equations. In Information processing 71 (Proc. IFIP Congr., Ljubljana, 1971), Vol. 2: Applications, pages 1206-1213. North-Holland, Amsterdam, 1972.

77. X. Yang, J. J. Feng, C. Liu, and J. Shen. Numerical simulations of jet pinching-off and drop formation using an energetic variational phase-field method. J. Comput. Phys., 218:417-428, 2006.

78. P. Yue, J. J. Feng, C. Liu, and J. Shen. A diffuse-interface method for simulating two-phase flows of complex fluids. J. Fluid Mech, 515:293-317, 2004. 